\title{
VLT observations of the asymmetric Etched Hourglass Nebula, MyCn 18
}

\author{
N. Clyne ${ }^{1}$, M. P. Redman ${ }^{1}$, M. Lloyd ${ }^{2}$, M. Matsuura ${ }^{3}$, N. Singh ${ }^{1}$, and J. Meaburn ${ }^{2}$ \\ ${ }^{1}$ Centre for Astronomy, School of Physics, National University of Ireland Galway, University Road, Galway, Ireland \\ e-mail: [n.clyne1; matt.redman]@nuigalway.ie \\ 2 Jodrell Bank Centre for Astrophysics, School of Physics and Astronomy, University of Manchester, Oxford Road, \\ Manchester M13 9PL, UK \\ 3 Department of Physics and Astronomy, University College London, Gower Street, London WC1E 6BT, UK
}

Received 21 June 2013 / Accepted 1 July 2014

\begin{abstract}
Context. The mechanisms that form extreme bipolar planetary nebulae remain unclear.

Aims. The physical properties, structure, and dynamics of the bipolar planetary nebula, MyCn 18, are investigated in detail with the aim of understanding the shaping mechanism and evolutionary history of this object.

Methods. VLT infrared images, VLT ISAAC infrared spectra, and long-slit optical Echelle spectra are used to investigate MyCn 18. Morpho-kinematic modelling was used to firmly constrain the structure and kinematics of the source. A timescale analysis was used to determine the kinematical age of the nebula and its main components.

Results. A spectroscopic study of MyCn 18's central and offset region reveals the detailed make-up of its nebular composition. Molecular hydrogen, atomic helium, and Bracket gamma emission are detected from the central regions of MyCn 18. ISAAC spectra from a slit position along the narrow waist of the nebula demonstrate that the ionised gas resides closer to the centre of the nebula than the molecular emission. A final reconstructed 3D model of $\mathrm{MyCn} 18$ was generated, which provides kinematical information on the expansion velocity of its nebular components by means of position-velocity (P-V) arrays. A kinematical age of the nebula and its components were obtained by the P-V arrays and timescale analysis.

Conclusions. The structure and kinematics of $\mathrm{MyCn} 18$ are better understood using an interactive 3D modelling tool called SHAPE. A dimensional and timescale analysis of MyCn 18's major components provides a possible mechanism for the nebula's asymmetry. The putative central star is somewhat offset from the geometric centre of the nebula, which is thought to be the result of a binary system. We speculate that the engulfing and destruction of an exoplanet during the asymptotic giant branch phase may have been a key event in shaping MyCn 18 and generating of its hypersonic knotty outflow.
\end{abstract}

Key words. planetary nebulae: individual: MyCn 18 - binaries: general - stars: kinematics and dynamics - stars: winds, outflows circumstellar matter - infrared: general

\section{Introduction}

MyCn $18(\mathrm{RA}=13 \mathrm{~h} 39 \mathrm{~m} 35.07 \mathrm{~s}$, Dec $=-67 \mathrm{~d} 22 \mathrm{~m} 51.7 \mathrm{~s}(\mathrm{~J} 2000)$; PN G307.5-04.9; IRAS 13359-6707) is a beautiful example of an extremely tightly waisted bipolar planetary nebula (PN). The famous HST images of Sahai et al. (1999), who christened it the Etched Hourglass Nebula, show the remarkable degree of symmetry in the nebula and also the clear offset of the central star along the nebular minor axis. The question of how the diverse range of PNe shapes are produced from what is presumably a spherical asymptotic giant branch (AGB) star (Soker \& Harpaz 1992; Trammell et al. 1995; Sahai \& Trauger 1998; Sahai et al. 2009) continues to intrigue astronomers and the extreme case of MyCn 18 provides a stern test of any evolutionary model.

The tight-waisted appearance of MyCn 18 led Corradi \& Schwarz (1993) to compare it to two apparently similar symbiotic nebulae He2-104 and BI Cru. However, they concluded that, other than the striking morphological similarity, there was no other evidence pointing to MyCn 18 being a post-PN symbiotic nebula and that it should tentatively be classed as a young
PN, on the basis of its position in optical and near-IR diagnostic diagrams.

Bryce et al. (1997) discovered hypersonic $\left(\sim 500 \mathrm{~km} \mathrm{~s}^{-1}\right)$ knots situated on either side of the main hourglass nebula, approximately along the polar axis. It is interesting to note that these knots are travelling much faster than the expansion velocity of the hourglass shell $\left(\sim 50 \mathrm{~km} \mathrm{~s}^{-1}\right)$. Such speeds are similar to those found by Corradi \& Schwarz (1993) for the symbiotic nebulae He2-104 and BI Cru. O'Connor et al. (2000) demonstrated that the knots of MyCn 18 had a degree of point symmetry and that the radial speed of each knot increased linearly with its distance from the central star. They speculated that MyCn 18 might be morphologically related to the symbiotic nebulae.

The HST images of Sahai et al. (1999) revealed a wealth of structural detail in the main nebula, which appears to contain a second, inner, hourglass-shaped structure, as well as two central rings. Each of these structures has a slightly different geometric centre, none of which are coincident with the central star. Dayal et al. (2000) showed that the nebula is generally densitybounded except in the region of the hourglass waist. They also 
inferred that the etched arcs on the hourglass walls might indicate temporal variations in the mass-loss rate of the progenitor star. Bains et al. (2002) found a similar geometric offset between the nebula and the central star when observing at radio wavelengths. Their spectral index maps show that the nebular core appears to be more optically thick than the lobe regions at lower frequencies $(1384$ and $4800 \mathrm{MHz})$. The relatively low brightness temperatures were interpreted as a result of beam dilution of a clumpy structure inferred a filling factor of 0.15 at $1384 \mathrm{MHz}$. Their estimated total ionised mass of the nebula is in the region $0.2-0.8 M_{\odot}$ at a distance of $2.4 \mathrm{kpc}$.

Whitelock (1985) presented $J H K$ photometry for a sample of southern PNe and showed that the major source of the nearIR radiation from MyCn 18 is nebular in origin. Molecular hydrogen has been detected in many PNe (Hora \& Latter 1996; Kastner et al. 1998, 2000; Likkel et al. 2000; Davis et al. 2003; Aleman \& Gruenwald 2004; Eyermann \& Speck 2004; Matsuura \& Zijlstra 2005; Hora et al. 2005; Wang et al. 2006; Matsuura et al. 2007; Sellgren et al. 2008; Ramos-Larios et al. 2008; Tenenbaum et al. 2009), and given that MyCn 18 is believed to be ionisation-bounded at its waist, it is likely that any available molecular hydrogen will be found around this region. Sahai et al. (1999) pointed out that the expected $K$-band photospheric flux (derived from optical photometry) is about half of the observed value, indicating that the central star is probably surrounded by hot dust in the waist region.

The hourglass shape of $\mathrm{MyCn} 18$ is possibly formed by a fast, tenuous wind expanding within a more slowly expanding cloud, which has a higher density near its equatorial region than near its polar regions. This snow-plow-like process was first introduced by Kwok et al. (1978), as the interacting stellar winds (ISW) theory of nebular formation, and later as the generalised interacting stellar winds (GISW) model (Kwok 1982; Balick 1987). The HST images of MyCn 18 reveal both intricate patterns (etchings) along its nebular walls and weak emission with filamentary components just beyond the walls. MyCn 18 is a good example of an extreme bipolar PN with strong asymmetry or axi-symmetry. This bipolar shape might also be the result of the formation of a dense equatorial accretion/excretion disk or of high-velocity collimated outflows, as discussed by Sahai et al. (1999).

The mechanism for generating extreme deviations from spherical symmetry in planetary nebulae remains unsettled. Models and explanations range from single stars with particular magnetic fields arrangements (Chevalier \& Luo 1994; García-Segura et al. 1999; Pascoli \& Lahoche 2008; Vlemmings 2011, 2012; Jordan et al. 2012) to the view that almost all planetary nebulae are caused by binary companions (Yungelson et al. 1993; Soker \& Rappaport 2001; De Marco 2009; Jones et al. 2010, 2012; Douchin et al. 2012). The discovery that hot Jupiter exoplanets are common around stars means that large planetary mass companions can also be considered amongst the shaping mechanisms (Nordhaus \& Blackman 2006). Clearly it is of interest to search for a binary star in MyCn 18. The identification of the central ionising star in MyCn 18 appears quite firm though it is clearly noticeable that the star is significantly offset from the geometrical centre of the outer and inner hourglass, as identified by Sahai et al. (1999).

In order to further our understanding of this striking nebula we have carried out an infrared and spectroscopic study of MyCn 18 and used a 3D morpho-kinematic code called SHAPE ${ }^{1}$

\footnotetext{
1 SHAPE is accessible online at http://www. astrosen. unam.mx/ shape/
}

Table 1. Filters used for the NACO observations.

\begin{tabular}{lcccc}
\hline \hline Filter & $\lambda_{\mathrm{c}}(\mu \mathrm{m})$ & $\Delta \lambda(\mu \mathrm{m})$ & $t_{\exp }(\mathrm{s})$ & Resolution \\
\hline$J$ & 1.265 & 0.25 & 1100 & $0.20^{\prime \prime} \times 0.19^{\prime \prime}$ \\
$H$ & 1.66 & 0.33 & 1100 & $0.18^{\prime \prime} \times 0.11^{\prime \prime}$ \\
Ks & 2.18 & 0.35 & 1600 & $0.13^{\prime \prime} \times 0.11^{\prime \prime}$ \\
NB_2.122 & 2.122 & 0.022 & 3720 & $0.11^{\prime \prime} \times 0.09^{\prime \prime}$ \\
IB_2.30 & 2.30 & 0.06 & 540 & $0.13^{\prime \prime} \times 0.09^{\prime \prime}$ \\
\hline
\end{tabular}

Notes. A list of the filters used for the NACO observations, giving the $\lambda_{\mathrm{c}}$ and $\Delta \lambda$ for each filter together with the total on-target integration time and the resolution as measured from the psf of the star to the northeast of the nebular core.

(Steffen et al. 2011) to carefully reproduce the structural and spectral features of the nebula.

The paper is organised as follows: the observational data are presented in Sect. 2. Analyses of each topic are presented in Sect. 3. This section includes: infrared imaging, spectroscopic analysis, the modelling of optical kinematic data using SHAPE, a geometric dimensional analysis of the outer and inner regions of the nebula, and a timescale analysis of the nebula's three major components. A discussion and conclusions are found in Sects. 4 and 5, respectively.

\section{Observations and results}

\section{1. infrared images}

A series of infrared images of MyCn 18 were obtained in photometric conditions on 27 May 2003 using NAOS-CONICA (NACO: Rousset et al. 2003; Lenzen et al. 2003) on ESO's VLT(UT4) telescope. NACO was used with the S27 camera ( 0.027 arcsec per pixel and a field of view of $28 \times 28$ arcsec).

Continuum images were obtained using the $J, H$, and $K$ s broadband filters. The NB_2.12 filter was used to image the $\mathrm{H}_{2}$ (1-0) S(1) emission line and the IB_2.30 filter was used to look for evidence of CO $2.295 \mu \mathrm{m}$ emission. The filters, exposure times, and image resolution are detailed in Table 1.

The central star of MyCn $18\left(m_{\mathrm{v}}=14.9\right.$ and spectral type Of(C); as noted by Sahai et al. 1999 and Lee et al. 2007, respectively) was used as the AO reference star, with optical wavefront sensing via the VIS dichroic. Optical seeing at the observatory was 0.4-1.1 arcsec FWHM during the observed period. The observations were taken with the NACO_img_obs_FixedSkyOffset template using a 4 arcsec square jitter box and sky offset position 35 arcsec to the NE of the target position.

The data were reduced using a tailored version of the ORAC-DR pipeline (Cavanagh et al. 2008) invoking the UKIRT imaging recipes (Currie \& Cavanagh 2004), which had been adapted to account for NACO's tiny point-spread function. The final image from each filter was then aligned with the $K \mathrm{~s}$ image. These images are shown in Fig. 1, along with the narrowband [N II]6584 A HST image (Sahai et al. 1999), which has been regridded to the NACO pixel scale and aligned with the $K \mathrm{~s}$ image for consistency (the resolution of this image is about $0.09 \times 0.09$ arcsec). Note that neither the $\mathrm{H}_{2}$ nor the $\mathrm{CO}$ images have been continuum subtracted. The NB_2.122 filter, used for the $\mathrm{H}_{2}$ observations, produces a very irregular point spread function, and due to this irregularity we were unable to create a satisfactory continuum subtracted image. The stretch levels used for all images in both Figs. 1 and 2 are tabulated in Table 2. 
J

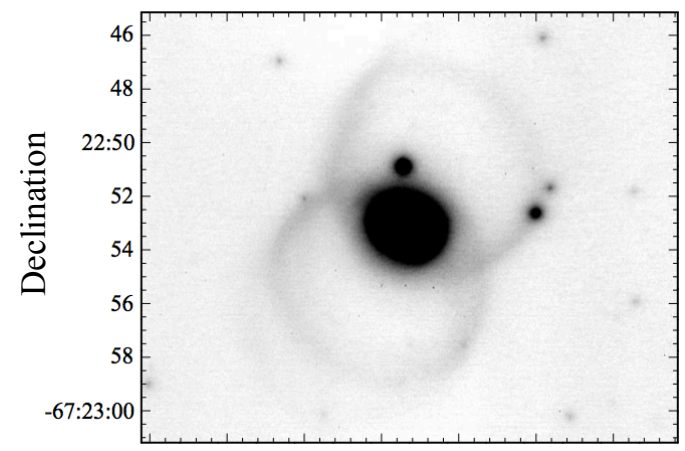

$\mathrm{H}_{2}$

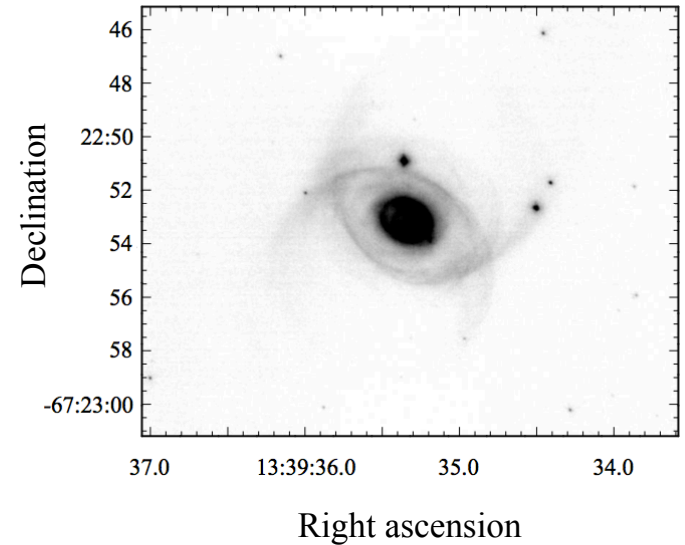

$\mathrm{H}$

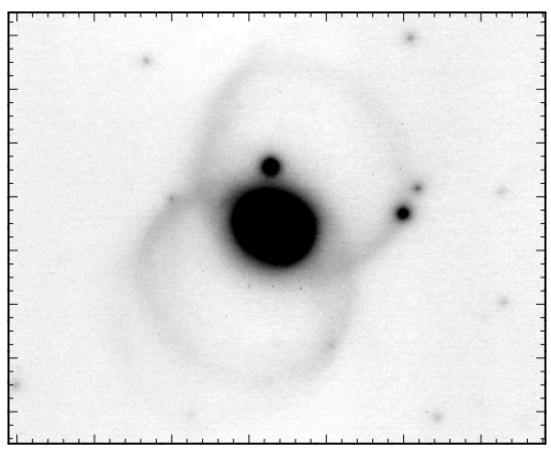

$\mathrm{CO}$

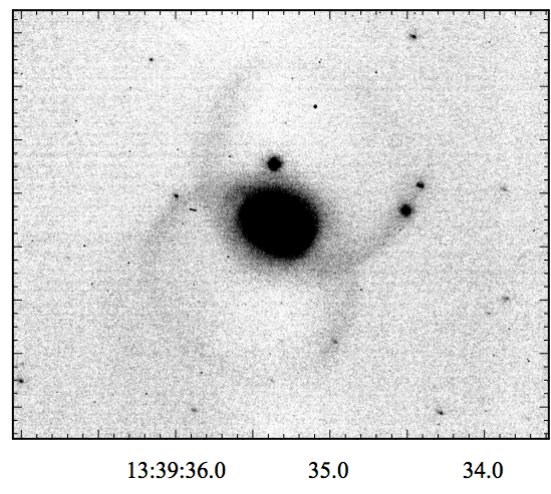

Right ascension
Ks

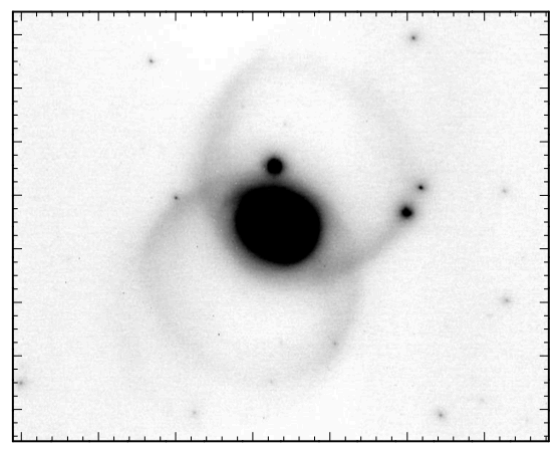

HST [NII]

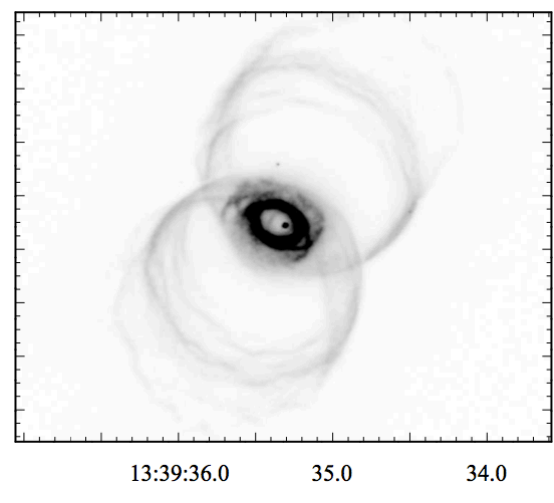

Right ascension

Fig. 1. Images of the bulk of MyCn 18's nebulosity. Presented are a series of linear greyscale infrared images obtained using the $J$, $H$, and $K$ s broadband filters, along with $\mathrm{H}_{2}$ and $\mathrm{CO}$ images using the NB_2.12 and IB_2.30 filters, respectively. An archival HST [N II]6584 $⿱$ A linear greyscale image is also included to compare and display the nebula's more distinct features.
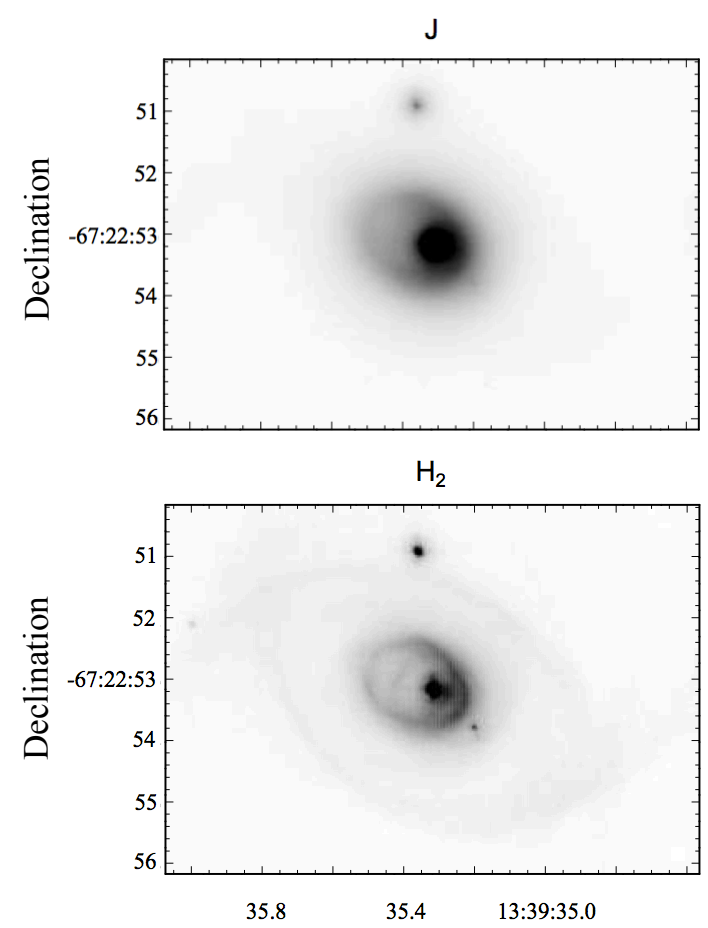

Right ascension

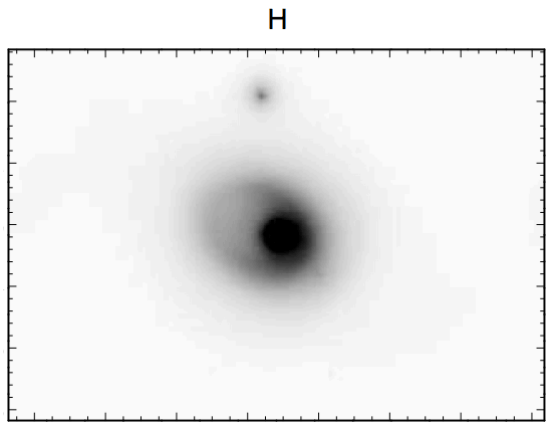

$\mathrm{CO}$

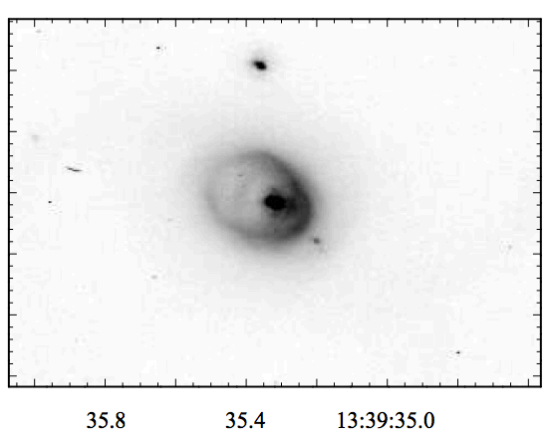

Right ascension

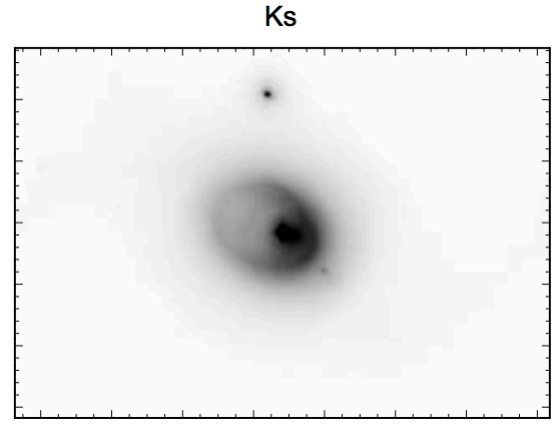

HST [NII]

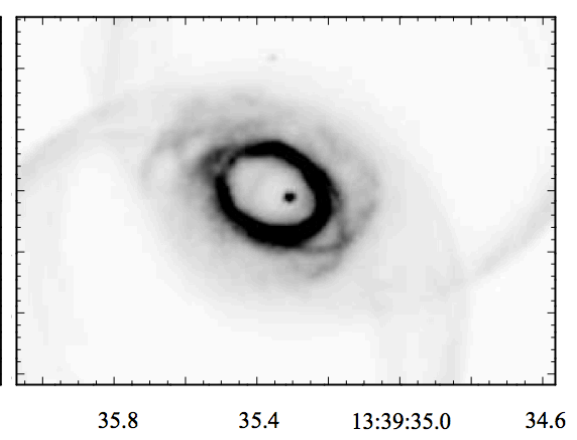

Right ascension

Fig. 2. Linear stretches of the images in Fig. 1. The central region of each image is displayed at a higher contrast linear scale to show the features of interest not apparent in Fig. 1. The stretches, in comparison to those images in Fig. 1, are as follows: $\mathrm{x} 10$ for $J, H, K \mathrm{~s}$ and $\mathrm{CO}, \mathrm{x} 4$ for $\mathrm{H}_{2}$, and $\mathrm{x} 2$ for [N II]6584 $\AA$. 
Table 2. Linear greyscale cut off levels used in Figs. 1 and 2.

\begin{tabular}{lcccccc}
\hline \hline Figures & $J$ & $H$ & $K \mathrm{~s}$ & $\mathrm{H}_{2}$ & $\mathrm{CO}$ & $\mathrm{N} \mathrm{II}$ \\
\hline 1 & $0-20$ & $0-20$ & $0-16$ & $0-15$ & $0-10$ & $0-800$ \\
2 & $0-200$ & $0-200$ & $0-160$ & $0-60$ & $0-100$ & $0-1600$ \\
\hline
\end{tabular}

Notes. Scaling was chosen to highlight the nebular features. The scales are in counts but are not flux calibrated. Individual frame exposure times were $10 \mathrm{~s}$ for $J, H$, and $K \mathrm{~s}$, and $60 \mathrm{~s}$ for $\mathrm{CO}$ and $\mathrm{H}_{2}$. The images shown in both figures are the combined and reduced images for each filter but with counts averaged to the exposure time for one image to give a better signal-to-noise.

The images in both Figs. 1 and 2 have been reduced in the standard way, except that no flux calibration has been applied. Each image represents a reduced mean frame uncorrected for exposure time. The standard stars GSPC P550-C $(\mathrm{RA}=10 \mathrm{~h} 33 \mathrm{~m} 51.9 \mathrm{~s}, \mathrm{Dec}=+04 \mathrm{~d} 49 \mathrm{~m} 05.0 \mathrm{~s})$ and GSPC S889-E $(\mathrm{RA}=22 \mathrm{~h} 02 \mathrm{~m} 05.73 \mathrm{~s}$, Dec $=-01 \mathrm{~d} 06 \mathrm{~m} 01.0 \mathrm{~s})$ were observed with the $J, H$, and $K$ s filters but we were unable to derive an accurate flux calibration, however, the results were broadly compatible with the overall calibrations found in Whitelock (1985).

The three continuum images in Fig. 1 all show faint relatively smooth emission from the edges of the main hourglass shell, together with a much brighter central region. The $\mathrm{H}_{2}$ image in contrast shows a much clearer thin, filamentary eye-shaped feature surrounding the bright inner region. Although the $\mathrm{H}_{2}$ image is not continuum subtracted, it is clear from the difference between it and the corresponding broadband $K$ s image that the eye-shaped feature is dominated by $\mathrm{H}_{2}$ line emission.

The bright central region of each image is reproduced in Fig. 2 at higher contrast to show the structure of the inner part of the nebula. Note that Figs. 1 and 2 are framed to progressively highlight the hourglass shape into the very central region of the eye. The broadband images again show a relatively smooth structure, dominated by an egg-shaped ring that is much brighter at its western, pointed end, just southwest of the central star. This ring was identified in the HST WFPC2 narrow-band optical images of Sahai et al. (1999) as ring 1. The central star is offset towards the narrow end of the ring, as noted by Sahai et al. (1999). An inner loop of emission corresponding to ring 2 is also apparent, particularly in the $\mathrm{H}_{2}$ image.

Figure 3 shows the central region of $\mathrm{MyCn} 18$ in (a) $\mathrm{H}_{2}$, identifying ring 1 and ring 2, and (b) in [N II]6584 A, revealing the nebula's inner hourglass. Ring 1 is defined as the waist of the outer hourglass and ring 2 appears to be part of the inner hourglass, which is a higher excitation ring due to its close proximity with the central star (Sahai et al. 1999).

\section{2. infrared spectroscopy}

Two-micron spectra of MyCn 18 were obtained with ISAAC (Moorwood et al. 1999) at the ESO Very Large Telescope (VLT) on 29 April 2004. An ISAAC short wavelength band was equipped with the Rockwell $1024 \times 1024$ array with pixel scale 0.145 arcsec in imaging mode and 0.147 arcsec in spectroscopic mode. The weather was clear, optical seeing varied between $0.92-1.37$ arcsec and seeing at the $K$-band was approximately 0.75 arcsec during the acquisition of the target. The slit was placed along the minor axis of the nebula at a PA of $62.45^{\circ}$ to the east (see Fig. 4) and the spatial scale was 0.15 arcsec per pixel. Low spectral resolution (LR) mode was used, and the slit width was 0.8 arcsec, resulting in a wavelength resolution

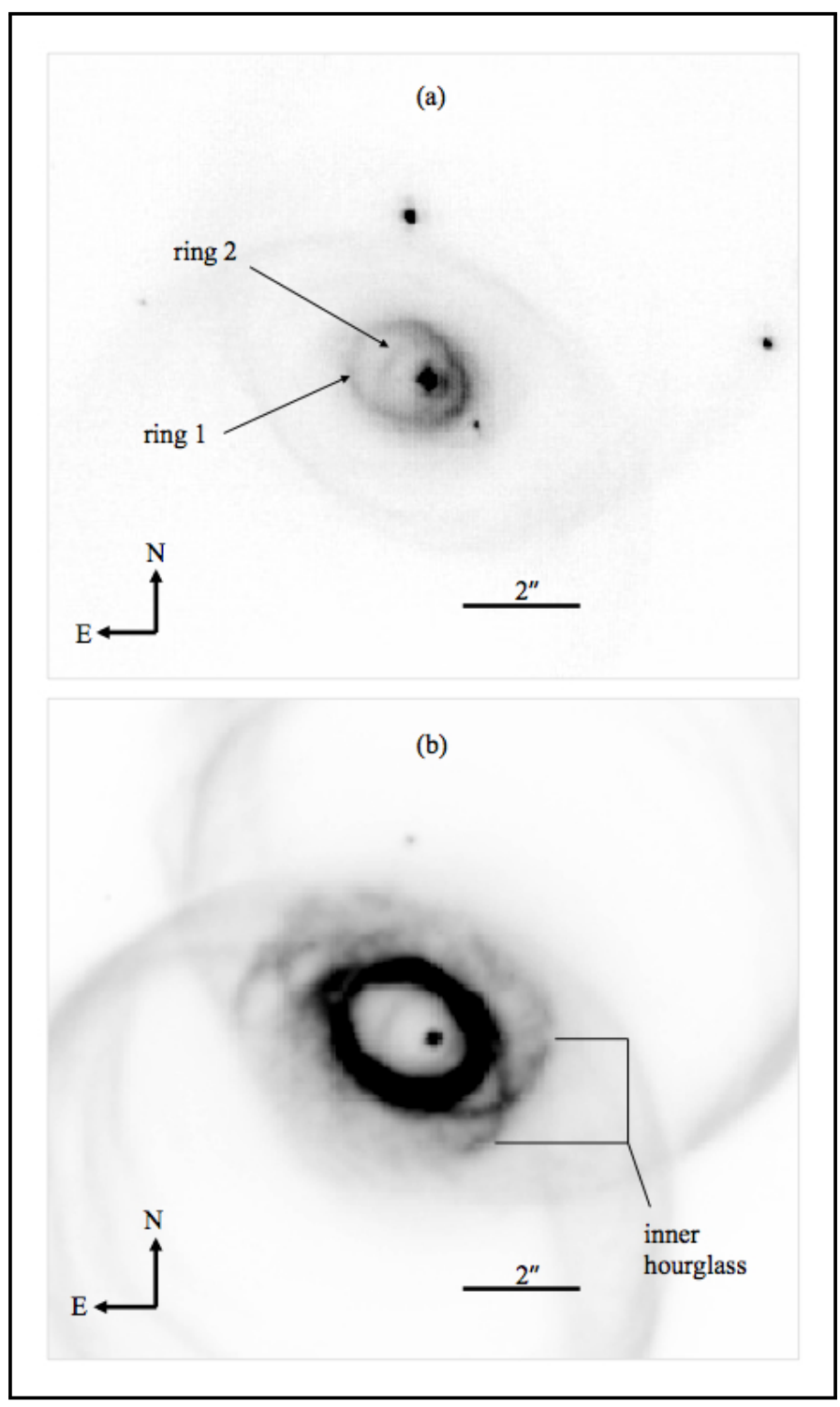

Fig. 3. Inner region of $\mathrm{MyCn} 18$. a) Image of ring 1 and ring 2 in $\mathrm{H}_{2}$; and b) the inner hourglass in HST [N II] $6584 \AA$.

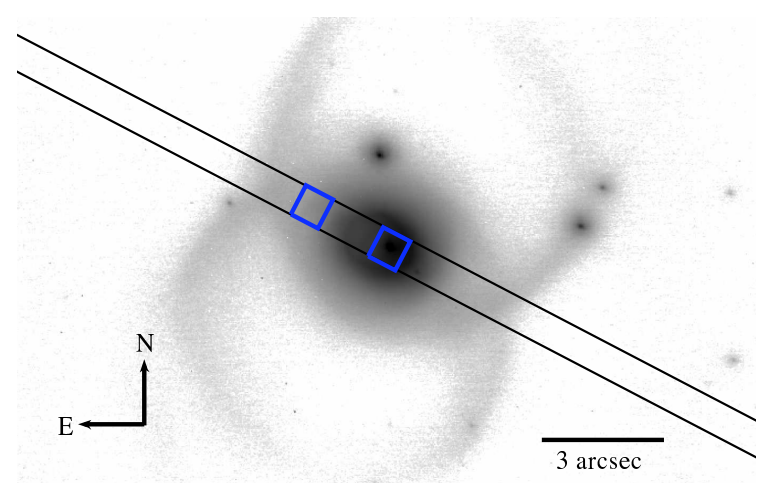

Fig. 4. Approximate ISAAC slit position displayed on NACO $K$ s image. Two boxes show the positions whose spectra have been displayed in Fig. 6.

$(R=\lambda / \Delta \lambda)$ of 750 . The telescope was nodded by 60 arcsec along the slit to cancel the sky background. Jitter was used along the slit to minimise the influence of hot pixels. An exposure 

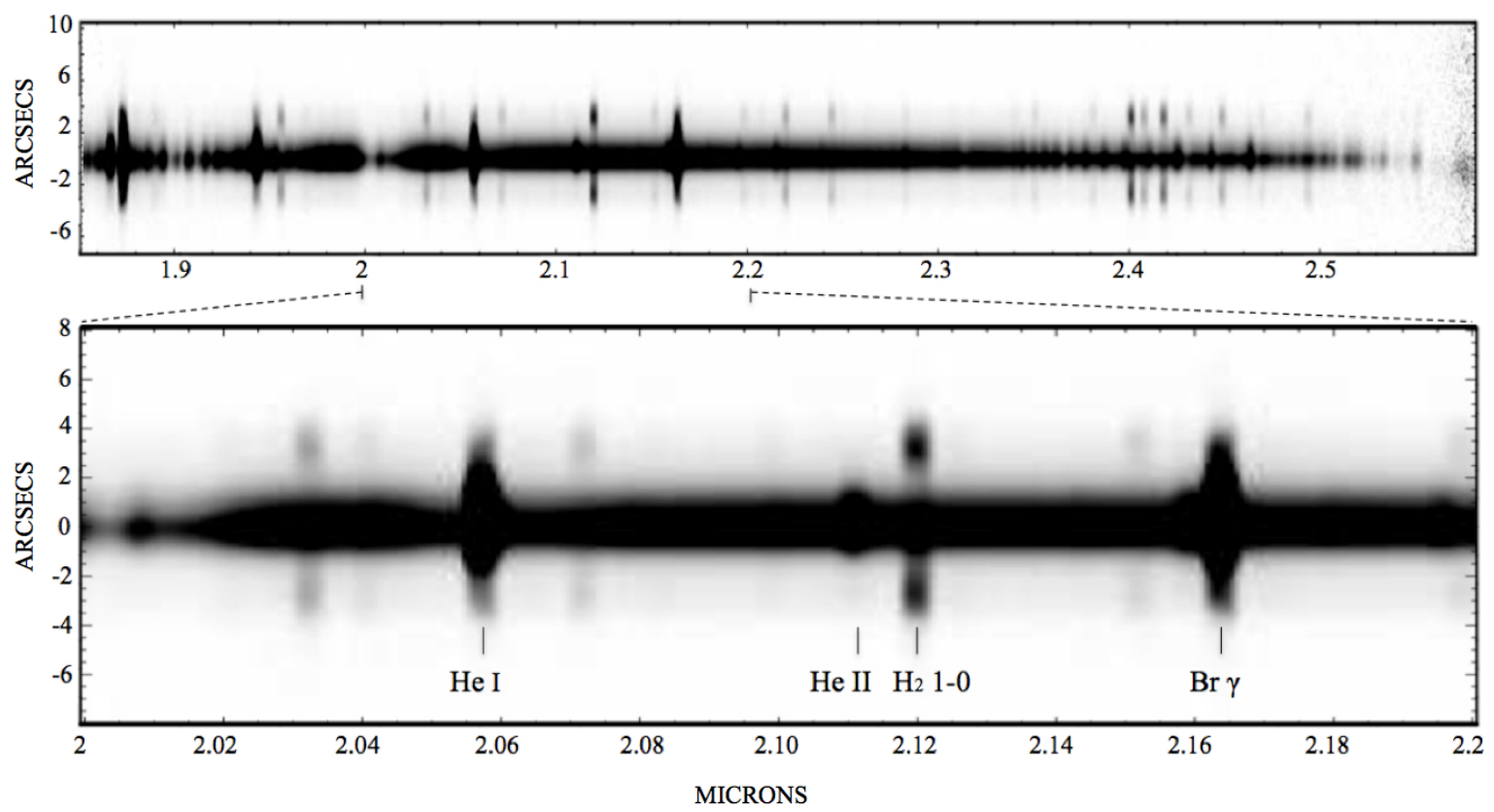

Fig. 5. A spatially resolved VLT ISAAC (outflow) spectrum. The spectrum, taken across the minor axis of the main hourglass shell, is shown at high contrast with the most prominent atomic and molecular emission lines visible. Two-micron spectrum (top), 2.25 arcsec from the central region along the nebula's minor axis, shows a plethora of strong $\mathrm{He}$ recombination and $\mathrm{H}_{2}$ vibrational lines. Bottom is an enlarged view of the region of this spectrum over the range 2-2.2 micron. More details of this spectrum are shown in Fig. 6

of twilight sky was used for flat-fielding. The exposure time was $120 \mathrm{~s} \times 16$ on source.

The approximate ISAAC slit location is shown in Fig. 4 and the orientations of the slit are from northeast to southwest, which follow from top to bottom in the figure. The two boxes across the ISAAC slit represent the regions of the centre and outflow spectrum. The data were reduced using IDL and the ECLIPSE package. The telluric standard was the G2V star Hip 053690; Pickles (1998) calculated the spectrum of this star. The wavelength calibration is based on exposures of an $\mathrm{Ar}+\mathrm{Xe}$ arc lamp with the same wavelength setting as the target observations. Additionally, OH skylines (Oliva \& Origlia 1992; Maihara et al. 1993; Rousselot et al. 2000) and telluric absorption lines (Hadrava 2006) were used for the fine adjustment of the wavelength calibration.

The 2D spectrum, shown at high contrast in Fig. 5, has been binned by 5 pixels along the slit length and indicates the most prominent atomic and molecular emission lines. The spectrum is seen to contain both continuum emission from the central region of $\mathrm{MyCn} 18$ and line emission from the surrounding nebular shell. A spatially extended nebular continuum is clearly shown to be about 2 arcsec in extent and slightly offset with respect to the nebula's geometric centre. The continuum emission has a faint extension to the east of the array. Two types of emission lines can be identified: one which is brightest in the core region and the other which is relatively faint in the core region but is brighter at each end, where the slit crosses the eastern and western edges of the nebula. This spectrum shows the spatial extent of $\mathrm{H}_{2}, \mathrm{He}$, and $\mathrm{Br} \gamma$ lines but is not corrected with telluric standard.

In Fig. 6, a $K$-band spectrum of MyCn 18 extracted from the central region is compared to a spectrum extracted from the nebular outflow region. The identifications of representative lines are also marked in the figure. In the central region, continuum is dominant, and both the central star and free-free and bound-free contribute to this continuum, as found in the images. This continuum contributes to approximately $8 \%$ of the total in-band flux in the $K \mathrm{~s}$-band. There are strong $\mathrm{H}(\mathrm{Br} \gamma$ and $\mathrm{Br} \delta$ ) and $\mathrm{He}$ recombination lines. Two $\mathrm{H}_{2}$ lines can be identified at $1.95 \mu \mathrm{m} 1-0 \mathrm{~S}(3)$ and $2.12 \mu \mathrm{m} \mathrm{1-0} \mathrm{S(1).} \mathrm{The} \mathrm{identifications}$ of the lines beyond $2.35 \mu \mathrm{m}$ are uncertain, but some $\mathrm{H}_{2}$ lines are present. In contrast, a forest of $\mathrm{H}_{2}$ lines is found in the outflow region, in addition to the $\mathrm{H}$ and $\mathrm{He}$ recombination lines.

The spectra shown in Figs. 5 and 6 demonstrate the spatial variations of the spectra across the object. The spectrum at the central region shows a strong underlying continuum across $K$-band spectra, particularly below the $2.2 \mu \mathrm{m}$ wavelength. Strong $\mathrm{H}$ and $\mathrm{He}$ recombination lines are present in this region but $\mathrm{H}_{2}$ lines are undetected. In contrast, the spectrum at the offset position, near the inner hourglass, is line dominated with some continuum. Here, He recombination lines are as strong as $\mathrm{H}$ recombination lines, but notably, much stronger $\mathrm{H}_{2}$ lines are detected across this region. These spectra reveal the dominant contributors to the $\mathrm{H}_{2}$ image (from Fig. 1) taken by the NACO. In the central region, there is a bright core found in the NB_2.122 image but this is due to continuum rather than $\mathrm{H}_{2}$. This image shows clear bipolar shaped outflows, which outline the hourglass shape and this part is likely to be formed by $\mathrm{H}_{2}$ emission lines. $\mathrm{CO}$ has a band of lines near/at the $2.3 \mu \mathrm{m}$ line, which correspond to a large number of P- and R-branch vibrational-rotational transitions.

\subsection{Optical images and spectroscopy}

To aid our analysis of MyCn 18 we obtained optical images from the Hubble Legacy Archive ${ }^{2}$ and ESO Archive, as well as MES and EMMI long-slit spectroscopic data.

2 Based on observations made with the NASA/ESA Hubble Space Telescope, obtained from the data archive at the Space Telescope Institute. STScI is operated by the association of Universities for Research in Astronomy, Inc. under the NASA contract NAS 5-26555. 

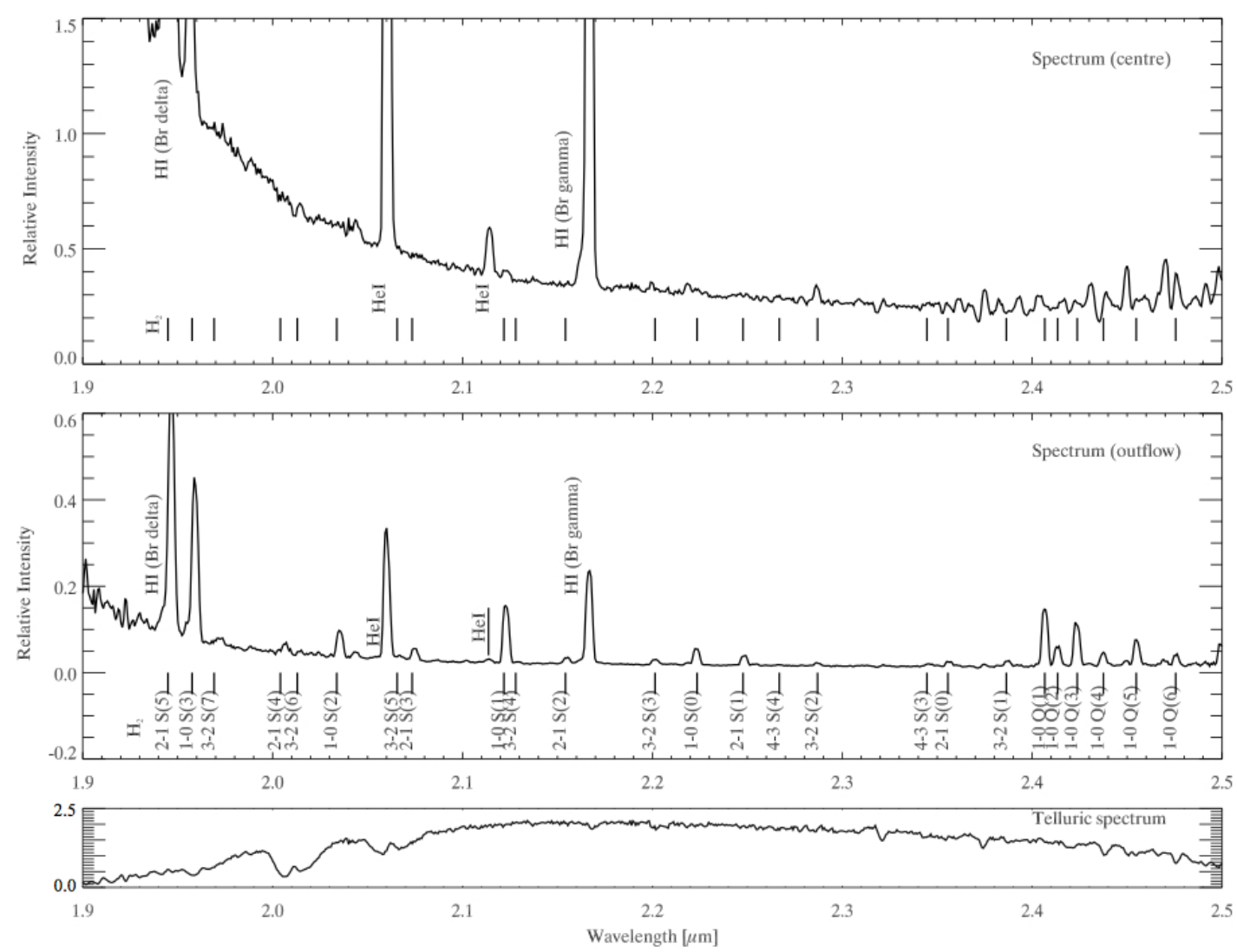

Fig. 6. Two-micron spectra of MyCn 18. Spectra for the central (top) 0.75 and offset (middle) 2.25 arcsec region are illustrated. Major H I and $\mathrm{He}$ II lines are indicated, and the expected positions of $\mathrm{H}_{2}$ lines are marked. The bottom panel shows the atmospheric features.

Position-velocity (P-V) arrays of [N II]6584 ̊ line profiles from long-slit spectra were obtained along the major and minor axis of MyCn 18. These long-slit spectra were not flux calibrated and they have not been relatively calibrated either because we are primarily interested in the kinematics. Both sets of spectra are displayed with a linear scale.

The major nebula axis long-slit spectra were taken with the Anglo-Australian telescope (AAT) using the Manchester Echelle Spectrometer (MES; Meaburn et al. 1984) between 26 and 28 March 1999. A slit width of $150 \mu \mathrm{m}$, which is equivalent to 1 arcsec, was used and limits the resolution in the spectral direction to be $11 \mathrm{~km} \mathrm{~s}^{-1}$. In the spatial direction, seeing was about 1 arcsec. The minor nebula axis long-slit spectra were obtained using ESO's Multi-Mode Instrument (EMMI; Dekker et al. 1986), which was attached to the ESO New Technology Telescope (NTT), and spectra were taken on 23 March 1994. The seeing conditions are the same for those of the AAT, described above. For additional details on the MES and EMMI spectra, see O'Connor et al. (2000) and Corradi \& Schwarz (1993), respectively.

\section{Analysis}

\subsection{Infrared images}

The images were analysed, using IRAF ${ }^{3}$, for possible companion stars to the nebula's primary. There is significant extinction and diffuse continuum emission to the centre of $\mathrm{MyCn} 18$

\footnotetext{
3 IRAF is distributed by the National Optical Astronomy Observatories, which are operated by the Association of Universities for Research in Astronomy, Inc., under cooperative agreement with the National Science Foundation.
}

(Bryce et al. 1997), which makes it difficult to resolve any close binary companions to the primary star. We believe the star located just SW of the central star in Fig. 3a has not been observed before. However, this star appears unlikely to be a candidate for association with the primary of MyCn 18 since it lies $\sim 0.9$ arc$\mathrm{sec}(\sim 2900 \mathrm{AU}$ for a $3.2 \mathrm{kpc}$ nebula distance) from the central star. Given that there are two other stars in the field of Fig. 3 then this newly observed star is also probably a field star; even if it were a companion, at this distance from the central star, it would have no direct dynamical effect on the shaping of the nebula.

Infrared contouring was later performed in and around the central region to search for even closer binary candidates. The astronomical data processing software STARLINK (Disney \& Wallace 1982) and its KAPPA (Currie \& Berry 2000) package were used to carry out the contouring. The reduced images in the $H$ and $K \mathrm{~s}$ bands, as well as that in $\mathrm{H}_{2}$, revealed some non-circular extension from the central star (see Fig. 3a). At first we thought this might be the result of a very close binary star, however, this non-point source appearance could be due to either (i) the aligning and co-adding of the images; (ii) poor psf as a result of adaptive optics; (iii) knotty emission from a recent ejection event; or (iv) a line of sight star. Due to the large intensity of continuum surrounding the central star, it is not presently possible to distinguish further between these possibilities. Further high resolution observations of the central region would be required to reveal the nature of this source.

\subsection{Spectroscopic analysis}

There are prominent atomic recombination lines from the central region extending outwards beyond the main continuum band. 


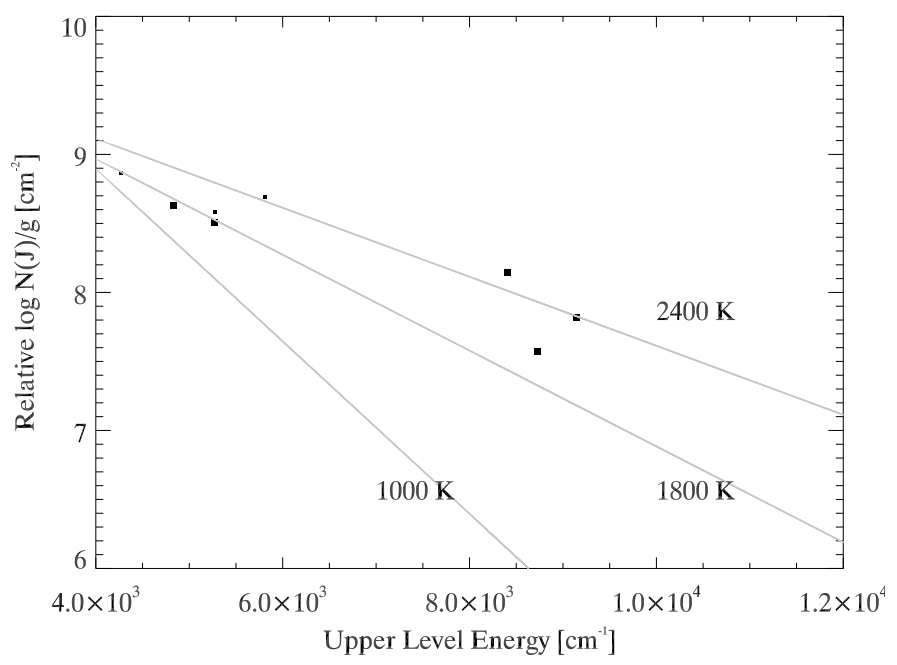

Fig. 7. An energy diagram of $\mathrm{H}_{2}$ at 2.25 arcsec to the northeast of the central region. Squares show the measured line intensities and the lines show expected $\mathrm{H}_{2}$ line intensities for a given temperature. The slopes of the lines indicate the temperature of the $\mathrm{H}_{2}$ and shows the modelled energy distributions for $1000 \mathrm{~K}, 1800 \mathrm{~K}$, and $2400 \mathrm{~K}$, where the vertical scales are arbitrary. The $\mathrm{H}_{2}$ excitation temperature is above $1800 \mathrm{~K}$.

The $\mathrm{H}_{2}$ lines are clearly different in spatial extent and peak at about \pm 3 arcsec from the star. Comparing this spatial cut to the images, it can be seen that the $\mathrm{H}_{2}$ emission comes from the cusps of the lenticular $\mathrm{H}_{2}$ feature, which is most likely the line-of-sight overlap of the two lobes of the hourglass. Sahai et al. (1999) noted that the expected $K$-band flux (from the star) is about half the level observed by Whitelock (1985) and suggested this may indicate the presence of hot dust in the equatorial region. Our spectra show that this excess emission can be explained instead by the strong free-free and atomic line emission from the compact ionised zone (Dayal et al. 1997; Koller \& Kimeswenger 2000; Volk 2003; Ramos-Larios et al. 2012a). Silicates typically have a dust condensation temperature of $\sim 1000 \mathrm{~K}$, therefore, the peak of the dust emission would appear at a longer wavelength $(2.9 \mu \mathrm{m})$ than what is observed in the spectra, which is actually $<1.9 \mu \mathrm{m}$. According to Corradi \& Schwarz (1993), MyCn 18 peaks at about $60 \mu \mathrm{m}(\sim 48 \mathrm{~K})$ and shows a typical double hump from its spectral energy distribution, indicating the presence of cool dust. Also, the light scattered by dust makes for an insignificant contribution to the nebula's central emission as noted by Sahai et al. (1999).

The resulting spectrum is the product of the nebula intensity gradient with a stratified ionisation structure, in which the least ionised layers are found furthest from the central star. The infrared spectroscopy shows clear ionisation stratification in that the $\mathrm{He}, \mathrm{H}$, and $\mathrm{H}_{2}$ lines are found progressively further from the central star into the putative surrounding molecular torus. The nebula is likely to be ionisation bounded in the equatorial direction but since the high-speed knots are ionised the nebula is more likely to be density bounded in the polar direction (Dayal et al. 2000; Bains et al. 2002).

Figure 7 shows the energy diagram of $\mathrm{H}_{2}$ lines in the outflow. The slopes, derived from the $\mathrm{H}_{2}$ lines, constrain the range of possible temperatures of the $\mathrm{H}_{2}$ emitting gas under the LTE assumption. This gives a temperature range of 1800-2400 K, though higher excitation lines may deviate from LTE (Matsuura et al. 2007). The $\mathrm{H}_{2}$ excitation temperature, estimated from

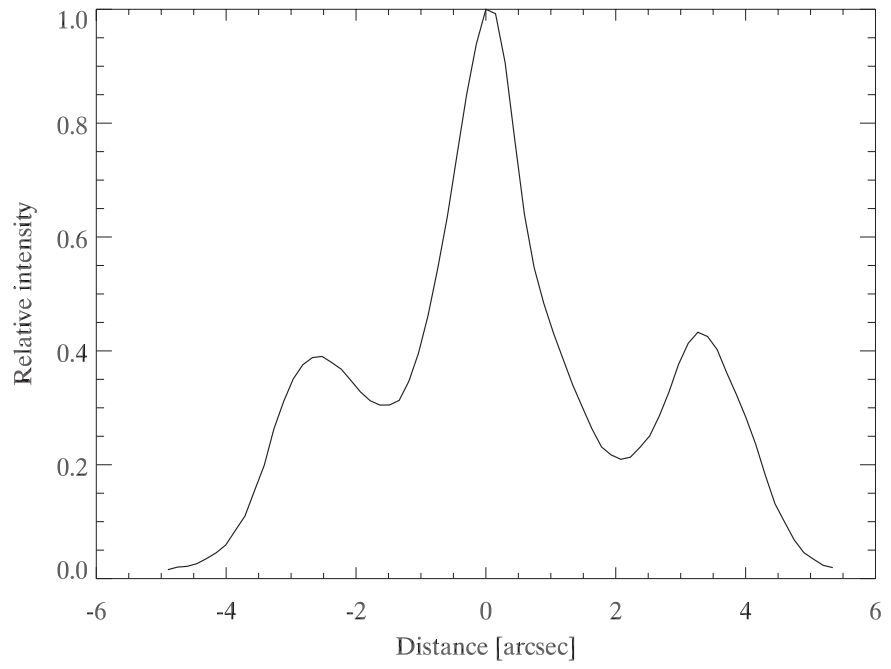

Fig. 8. A spatial cut through the $\mathrm{H}_{2}$ 1-0 $\mathrm{S}$ (1) line in Fig. 5 along the 2.122 micron pixel. The intensity distribution is shown and scaled at the peak.

rotation-vibrational lines, is as high as those estimated from similar lines of the Helix Nebula (1800 K; Matsuura et al. 2007) and the centre of NGC 6302 (1845 K; Davis et al. 2003) but much higher than temperatures estimated from rotation lines (below 1000 K; Cox et al. 1998; Bernard-Salas \& Tielens 2005). It appears that rotation-vibrational temperatures are always higher near the central region of PNe. This might require an extra heating mechanism such as UV excitation (Henney et al. 2007) or shock excitation of $\mathrm{H}_{2}$ (Burton et al. 1992).

The $K$ s band image in Fig. 2, which is dominated by the nebular continuum, shows an extent of about 2 arcsec across the central region. The spatial extent of the nebular continuum can also be readily seen in Fig. 5. Visually, it can be seen that the $\mathrm{H}_{2}$ 1-0 line emission (at $2.12 \mu \mathrm{m}$ ) is found closer to the continuum in the negative spatial (SW) direction than the positive spatial (NE) direction, as shown in Fig. 8. This corresponds with the asymmetry seen in the continuum images of Fig. 2.

\subsection{Geometric and timescale analysis of MyCn 18's nebular structures}

The nebula has noticeable deviations from axial symmetry as illustrated in Fig. 9. The figure shows the relative offsets of the various features within the nebula. As shown, the central star is not centred on the nebula's major axis and is clearly offset along the minor axis. The star is also offset from the centre of ring 1 and is closer to the centre of ring 2 (see Fig. 3a). A close observation of the inner hourglass shows a slight tilt of its structure with respect to the outer hourglass. The image on the right of Fig. 9 shows the relative offsets of the inner hourglass, the nebular waist (ring 1), and the central star with respect to the nebula's geometric centre. In the case of the central star, it remains unclear as to why it is offset to the west of the nebular waist. An explanation of this offset may involve a binary companion.

In relation to $\mathrm{MyCn} 18$ 's hypersonic knotty outflow, shown in Fig. 10, the paths taken by the knots are not symmetric about the axis of symmetry of the nebula's main hourglass (O'Connor et al. 2000). A closer inspection illustrates the scattering of the knots, with the northern cluster of knots found 


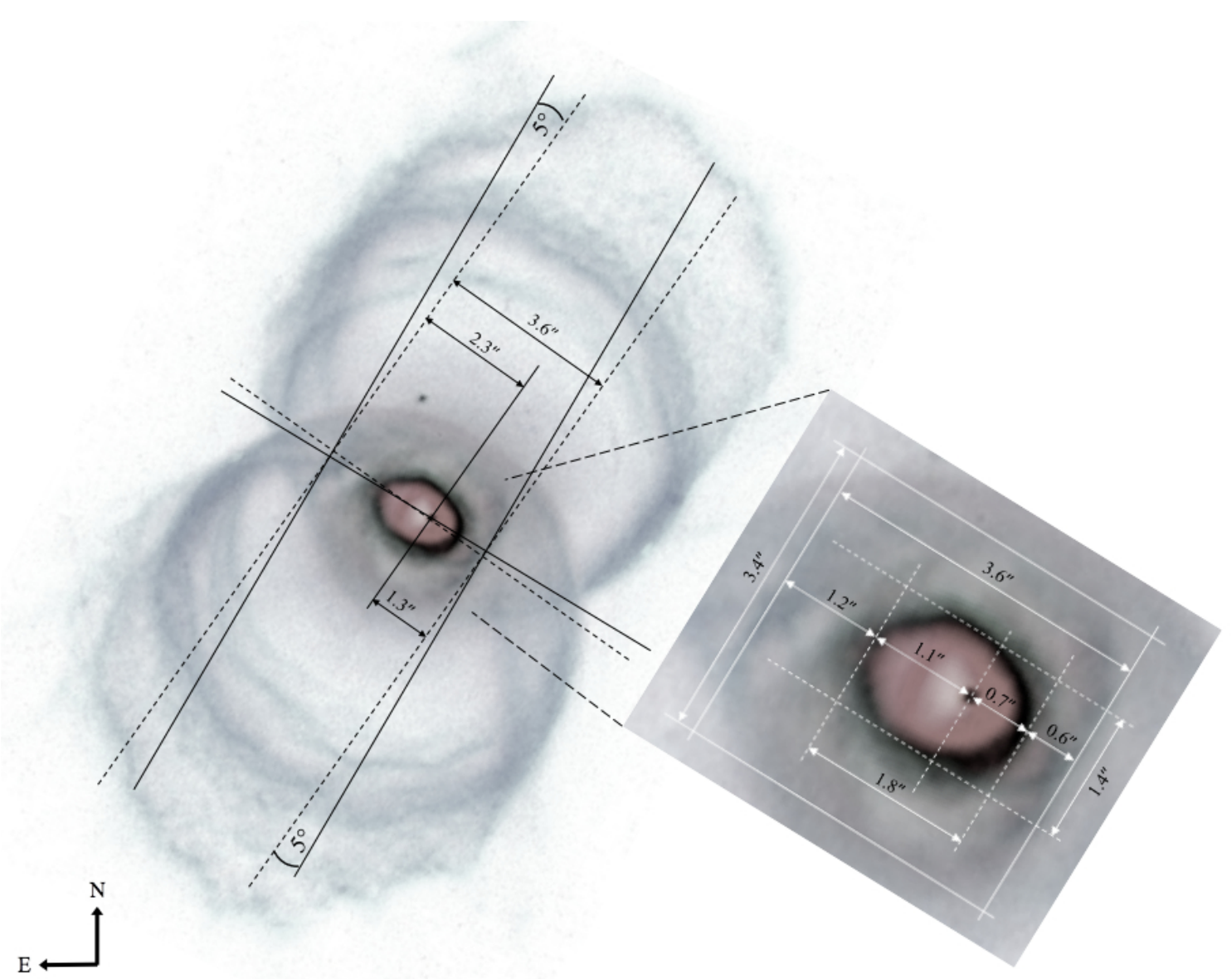

Fig. 9. An HST [N II]6584 $\AA$ image of MyCn 18. Shown is a geometric dimensional analysis of its inner and outer regions. The asymmetry of the nebula is obvious from the figure, with the inner hourglass being offset and slightly tilted $\left(\approx 5^{\circ}\right)$ with respect to the main hourglass shell and the central star is offset from the nebula's geometric centre.
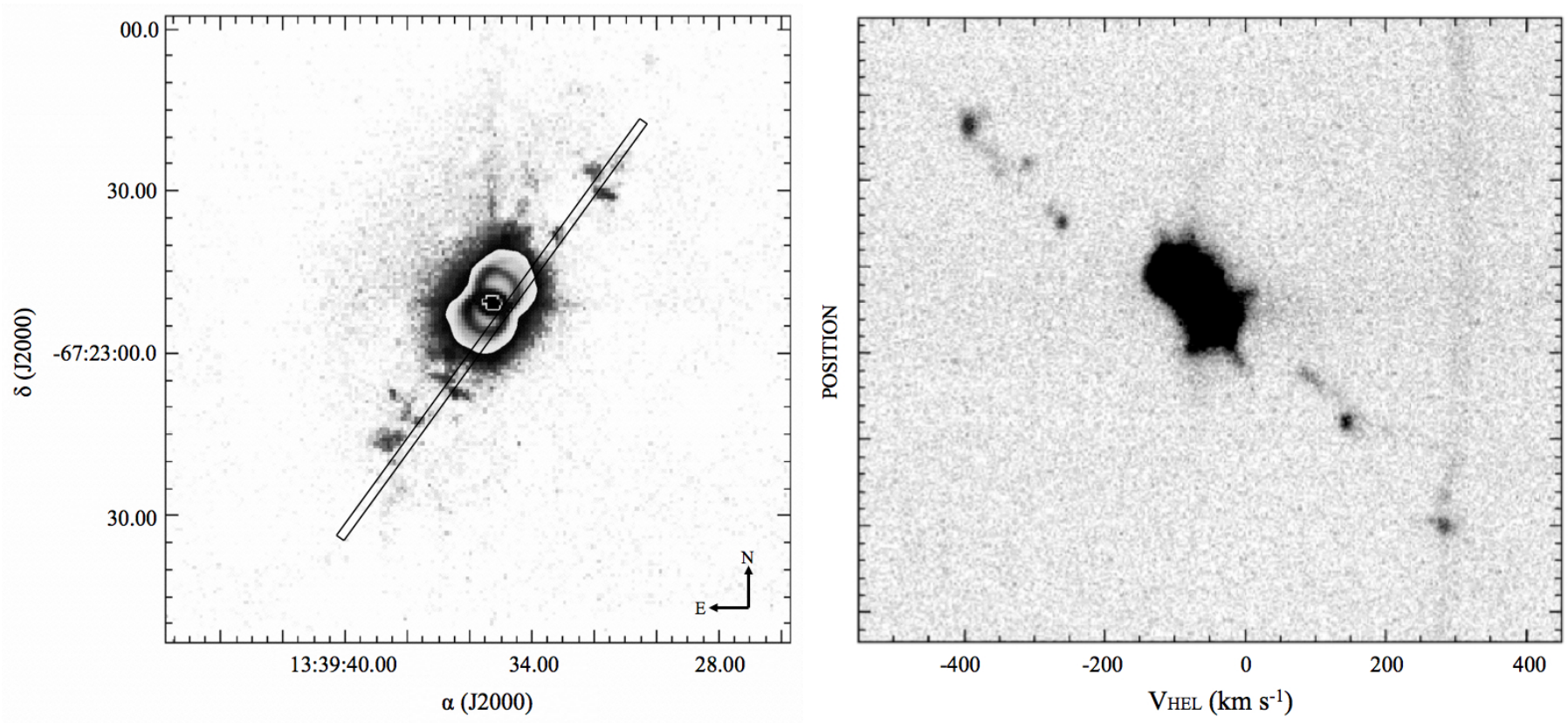

Fig. 10. Images of the hypersonic knotty outflow of MyCn 18. Left: a continuum subtracted image of MyCn 18 revealing the relative positions of the high-speed knots. Right: a P-V array for a long-slit placed down the nebula's major axis and through various knots, located north and south of the nebular lobes. The slit position shown on the left is a rough estimate of that used to produce the P-V array on the right. The knots south of the nebula (and through the slit) in the continuum subtracted image present the knots in the top left of the P-V array, and conversely, the knots north of the nebula present those in the bottom right. Both images in the figure are adopted from O'Connor et al. (2000). 
predominantly to the right of the main hourglass' axis of symmetry, and conversely, the southern cluster positioned to the left of this axis. The knots appear better aligned with the inner hourglass' axis of symmetry, thus supporting the hypothesis of a connection between these components.

The morphology and kinematics of MyCn 18 were better understood by applying timescales to each of its major components. These components include the main hourglass structure, the inner hourglass, and the high-speed interstellar knots. A distance of $2.4 \mathrm{kpc}$ is routinely quoted for $\mathrm{MyCn} \mathrm{18,} \mathrm{which} \mathrm{is} \mathrm{in-}$ ferred from a statistical method by Shklovsky (Schwarz et al. 1992). A more recent re-analysis of this method by Stanghellini et al. (2008) finds in particular that values derived in this way, by Corradi \& Schwarz (1993), underestimate the distance for butterfly type planetary nebulae. They find a value for the distance to be $3.2 \mathrm{kpc}$. Other distances quoted in the literature range from $1 \mathrm{kpc}$ to $3 \mathrm{kpc}$.

The fastest knots are travelling with speeds of $\sim 500 \mathrm{~km} \mathrm{~s}^{-1}$ with the kinematical age of the fastest knot being $\sim 1250 \mathrm{yr}$ (Bryce et al. 1997; O'Connor et al. 2000). Dayal et al. (2000) presented details of the velocities and corresponding timescales along different latitudes of the main hourglass and found the kinematical age of the nebula to be $\sim 2500 \mathrm{yr}$.

While the radius, $R$, of the nebula will vary linearly using the adopted distance, the expansion velocity follows a weaker power law in $R\left(V_{\exp } \propto R^{(0.6 \pm 0.4)}\right.$ : Dayal et al. 2000), hence the nebula expansion timescale is less sensitive than the estimated timescale of the ballistic ejection of the hypersonic knots. Our value for the kinematical age of the fastest knot, using the $3.2 \mathrm{kpc}$ distance, is $\sim 1650 \mathrm{yr}$. With regards to the kinematical age of the nebula, the value obtained was $\sim 2700 \mathrm{yr}$.

The shape and structure of the inner hourglass appears similar to that of the main hourglass, which suggests that the mechanism that gave rise to the main hourglass might also be the same for that of the inner hourglass. Assuming this theory, the inner hourglass should follow a similar velocity law $\left(V_{\exp } \propto R^{0.6 \pm 0.4}\right)$ to that of the main hourglass. In this case, the expansion velocity of the inner hourglass would increase with increasing latitude along its walls, and is estimated to have an expansion velocity of $30 \pm 5 \mathrm{~km} \mathrm{~s}^{-1}$ according to the synthetic P-V arrays generated from our SHAPE model. This corresponds to a timescale of $1750 \pm 250 \mathrm{yr}$. The interstellar knots have a similar kinematic age, thus implying that there might be a connection between them and the nebula's inner hourglass.

\subsection{Morpho-kinematic modelling of MyCn 18: SHAPE analysis}

While the hourglass morphology appears obvious, the significant inclination of $\mathrm{MyCn} 18$ means that the structure and dynamics of the crucial region around the central star is not clear. Following the treatment of Hubble 12 in Vaytet et al (2009), the astrophysical modelling program SHAPE (version 4.51) was used to create the morpho-kinematics of $\mathrm{MyCn} 18$ that were characterised by Bryce et al. (1997); Sahai et al. (1999); Dayal et al. (2000); O'Connor et al. (2000).

SHAPE is a software package that allows for the quick construction of many structures in $3 \mathrm{D}$ and comparing these results with observed data assists in the interpretation of the object of interest. The geometry of each structure can be easily customised by means of a 3D module and can be done either analytically or interactively. The application of a velocity field and emission properties to such a 3D structure model can allow for the generation of $2 \mathrm{D}$ rendered images and $\mathrm{P}-\mathrm{V}$ arrays, channel maps, light curves, and 1D spectral line profiles (Steffen et al. 2011). Planetary nebulae such as NGC 2392 (García-Díaz et al. 2012), IC 418 (Ramos-Larios et al. 2012b), HaTr 4 (Tyndall et al. 2012), NGC 7026 (Clark et al. 2013) and Kn 26 (Guerrero et al. 2013) are all interesting examples that have recently been modelled using SHAPE.

A morpho-kinematic model of $\mathrm{MyCn} 18$ was developed to allow for the reconstruction of its nebular morphology. Our aim, using SHAPE, involved creating a 3D shape resembling the nebula using observed P-V arrays (optical long-slit spectra), which are compared and matched up with synthetic P-V arrays generated from our model.

Development of the model involved using three main structures; a cylinder (for the outer hourglass), a sphere (for the inner hourglass), and a torus (central "eye" region). A cylinder, with a line type and a segment value of 30 (same in each structure), was used for the outer hourglass shell. A squeeze geometry modifier was then applied to the cylinder to give an hourglass shape with a tight waist of $1.8^{\prime \prime}$. A velocity modifier, which is a velocity field as a function of position, was also applied to help determine the kinematics of the outer hourglass shell. The vector field of this velocity modifier was set to give a radial Hubble expansion of $0 \mathrm{~km} \mathrm{~s}^{-1}$ at the centre to $70 \mathrm{~km} \mathrm{~s}^{-1}$ at the poles using a radial power law. The velocity function is given by $|f|(r)$ $=A+B\left(r / r_{0}\right)+C\left(r / r_{0}\right)^{D}$. The $r$ (initial arbitrary radius assigned to the main hourglass structure) parameter is the only constant with a value of 100 , whereas all other parameters are variables. The $r_{0}$ parameter is used to give the rate of change of velocity with respect to the radius $r$ and has a value of 15. Parameters $A$ and $B$ are 0 , whereas $C$ and $D$ are 2.2 and 1.82 respectively. Density modifiers, which set the spatial distribution of density, were used to give the arc-like etchings/rings on the outer hourglass shell. An arbitrary density scaling factor was used where the various structures were modified. A scaling factor of 1.0 was chosen for the main outer hourglass structure. The etchings/arcs, on each lobe, varied in density from 2.0 to 5.0. Two separate density modifiers were applied to the nebular waist (torus structure representing both ring 1 and ring 2). The first of these modifiers varied from 10 to 15 while the other (darkest region of the eye) varied from 15 to 20 . All density values were chosen empirically by reference to the HST imagery of MyCn 18 from Sahai et al. (1999). Other modifiers such as shell thickness, size, and custom vertex were used to complete the outer hourglass structure. It should also be noted that the squeeze and density modifiers were set interactively, whereas the rest were set analytically. The inner hourglass was developed using a sphere. Again, a squeeze modifier was applied to give its hourglass shape, and a velocity modifier was set to give a radial expansion from $0 \mathrm{~km} \mathrm{~s}^{-1}$ at the centre to $30 \mathrm{~km} \mathrm{~s}^{-1}$ at the poles. The only different modifiers used for this structure were a translation modifier to allow for the offset $\left(0.3^{\prime \prime}\right)$ of the structure and a rotation modifier to give the slight rotation $\left(5^{\circ}\right)$ of the inner hourglass with respect to the outer hourglass. The central eye region (nebular waist) of the nebula was constructed using a torus. The only modifiers not used for this structure were the squeeze, translation, and rotation modifiers. The expansion velocity is radially from $0 \mathrm{~km} \mathrm{~s}^{-1}$ at its centre to $10 \mathrm{~km} \mathrm{~s}^{-1}$ at its edge. A density modifier was used to give the ring 2 feature, as shown in Fig. 3a. The value for the inclination of each structure and their features is set to $38^{\circ}$ on the $2 \mathrm{D}$ rendering module, which corresponds to $90^{\circ}-38^{\circ}=52^{\circ}$. See Table 3 for the parameters used to create the model.

The P-V arrays shown in Fig. 11 (AAT \& NTT arrays) and the associated P-V arrays from O'Connor et al. (2000) were used as the basis for the reconstruction process. Those observed 
Table 3. The parameters for the best-fit SHAPE model of MyCn 18.

\begin{tabular}{lc}
\hline \hline Parameter & Value \\
\hline Pole-to-pole length (along its major axis) & $18^{\prime \prime}$ \\
Nebular waist (along its minor axis) & $1.8^{\prime \prime}$ \\
Max lobe width (along its minor axis) & $8.5^{\prime \prime}$ \\
Shell thickness & $0.2^{\prime \prime}$ \\
Inclination (w.r.t. plane of sky) & $52^{\circ}$ \\
Systemic heliocentric radial velocity, $V_{\text {sys }}$ & $-71 \mathrm{~km} \mathrm{~s}^{-1}$ \\
Max velocity (outer hourglass) & $70 \mathrm{~km} \mathrm{~s}^{-1}$ \\
Max velocity (inner hourglass) & $30 \mathrm{~km} \mathrm{~s}^{-1}$ \\
Convolution (seeing) & $1.37^{\prime \prime}$ \\
\hline
\end{tabular}

Notes. The parameters for the velocity of the inner and outer hourglass varied until the observed and synthetic $\mathrm{P}-\mathrm{V}$ arrays resembled each other closely. The slit width used in the modelling is the same as that used for the observed data.

spectra from Fig. 11 are shown at two different contrasts to display the spectroscopic features of $\mathrm{MyCn} 18$. The stretches for the AAT spectra, from top to centre, are 0-60000 and 015000 counts respectively, and the stretches for the NTT spectra, from top to centre, are 0-40000 and 0-10000 counts, respectively. Also shown in Fig. 11 are synthetic P-V arrays, which were generated from the reconstructed model of MyCn 18 by means of a rendering module. The features of the synthetic $\mathrm{P}-\mathrm{V}$ arrays reveal more detail than those of the observed arrays because of the lesser seeing for the observed data on those given nights. It was for this reason that the P-V arrays from O'Connor et al. (2000) needed to be used also. The discrepancies in the observed data were brought out more clearly in the modelled arrays by varying the convolution (seeing) parameters on the rendering module of SHAPE. Figures 11 and 12 were colour-inverted and grey-scaled for clear presentation.

A systemic heliocentric velocity, $V_{\text {sys }}$, of $-71 \mathrm{~km} \mathrm{~s}^{-1}$ (O'Connor et al. 2000) was used as the central velocity in the synthetic P-V arrays. The brightness of the observed P-V arrays varies from low to high for a long-slit placed directly down the major axis of the nebula and across its minor axis. The MES arrays (major-axis arrays) show the lobed features, which are more visible in the higher brightness image. The same features are clearly displayed in the model slit 1 synthetic array with better seeing than the observed MES arrays. It is apparent that both the observed and synthetic arrays follow a similar pattern, which closely indicates that the shape of $\mathrm{MyCn} 18$ is an open ended, bilobed, bipolar nebula.

The rates of expansion (in $\mathrm{km} \mathrm{s}^{-1}$ ) from the geometric centre out to the end of each lobe and across the waist were determined from the synthetic arrays. Having applied the appropriate velocity components, using the SHAPE velocity modifiers, the velocity scale can be centred at $V_{\mathrm{HEL}}=-71 \mathrm{~km} \mathrm{~s}^{-1}$, the velocity defined with respect to the nebula's frame of reference in which the Sun is at rest. Two distinctly separate velocity features are present in the model slit-1 and MES arrays with the first around $0^{\prime \prime}$ offset from $V_{\mathrm{HEL}}=0$ to $-140 \mathrm{~km} \mathrm{~s}^{-1}$ and the other from $V_{\mathrm{HEL}}=-30$ to $-120 \mathrm{~km} \mathrm{~s}^{-1}$ for the model slit- 1 array. The MES arrays, however, do not show emission out to these velocities, therefore, the P-V arrays from O'Connor et al. (2000) (see Fig. 3) were used to help finalise the synthetic arrays. These velocities obtained closely resemble those found by O'Connor et al. (2000) for their observed data.

The finished model, shown in Fig. 12a as a 2D rendered image, has the same inclination (with the top, southeastern, half of the hourglass tilted towards us at an angle of $52^{\circ}$ with respect to the plane of the sky) and is closely scaled to that shown in Fig. 12b, which is an HST [N II]6584 ̊̊ image for comparison. It should also be noted that the seeing used for the $2 \mathrm{D}$ rendered image is 0.3 arcsec instead of the 1.37 arcsec used when generating the synthetic P-V arrays, and the structures of the observed P-V arrays are smeared due to the 1.37 arcsec seeing, which makes it difficult to get a precise measurement of the expansion.

\section{Discussion}

\subsection{Shaping mechanisms and offsets}

Although there is still much to be understood about the complexity of MyCn 18's asymmetry, we here propose explanations as to why this might be. Currently, the binary scenario is the favoured mechanism for the creation of bipolar planetary nebulae (Morris 1987; Han et al. 1995; Soker \& Rappaport 2000; Balick \& Frank 2002; De Marco 2009; Jones et al. 2010; Soker \& Kashi 2012). Companion stars may likely exist within the central eye region of MyCn 18, however, since they cannot be observed directly, their luminosity must be low in comparison to the neighbouring central star, and any continuum surrounding the central region would certainly obscure their light.

In relation to the offset of the central star, one proposed theory involves a variable accretion of matter onto the central white dwarf, resulting in a dwarf nova explosion that could have kicked the star off in one direction while producing the disrupted region of the eye in the opposite direction (see N-E region of ring 1 in Fig. 3b). Assuming momentum conservation of the ejecta+star system before and after the explosion, we determine an energy of $7.2 \times 10^{43} \mathrm{erg}$ for a velocity of $600 \mathrm{~km} \mathrm{~s}^{-1}$ (Bryce et al. 1997; O'Connor et al. 2000) and a mass of $10^{-5} M_{\odot}$ (Sahai et al. 1999) for the knotty ejecta. From Fig. 9, we see that the star is offset from ring 1 by $0.2^{\prime \prime}$, which corresponds to $640 \mathrm{AU}$ for a distance of $3.2 \mathrm{kpc}$, and the time taken for the star to move from the nebula's geometric centre to its current position is $1050 \mathrm{yr}$ (the difference between the age of the nebula and the knotty ejecta: $2700-1650 \mathrm{yr}$ ). Using these values, we find that the star would have to be moving at a velocity of $\sim 3 \mathrm{~km} \mathrm{~s}^{-1}$ over that period. For a $0.6 M_{\odot}$ white dwarf and a velocity of $3 \mathrm{~km} \mathrm{~s}^{-1}$, we find an energy of $5.4 \times 10^{43} \mathrm{erg}$, which closely resembles that of the energy for the ejecta plus star system.

The nebula's other prominent features are also offset with respect to the geometric centre. The offset, and even the creation, of the inner hourglass may be the result of the formation of the knots since its axis of symmetry appears to follow the path taken by the knots. Our calculation for the kinematical age of the inner hourglass corresponds closely to that of the knots, which further suggests a possible connection between both components.

The younger, high-speed knots might explain the truncated, open-ended lobes of the outer hourglass. At an earlier epoch, before the creation of the knots, the ends of the lobes may have been closed. Some time after, the high-speed ejection of the knots would have penetrated the lobed ends, giving rise to their truncation, thus suggesting the younger age of the knots. This penetration would have occurred when the outer hourglass was smaller than it is now, which could explain for the large opening angle of each lobe (see Sahai \& Trauger 1998).

Soker \& Kashi (2012) propose that the formation of bipolar lobes of some PNe and pre-PNe are the result of one or more intermediate-luminosity optical transient (ILOT) events. Jets created by the accretion of matter, from an AGB (or extremeAGB) star, onto a main-sequence companion are launched by an accretion disk, which in turn shapes the bipolar lobes. For 
N. Clyne et al.: VLT observations of the asymmetric Etched Hourglass Nebula, MyCn 18

AAT - MAJOR-AXIS

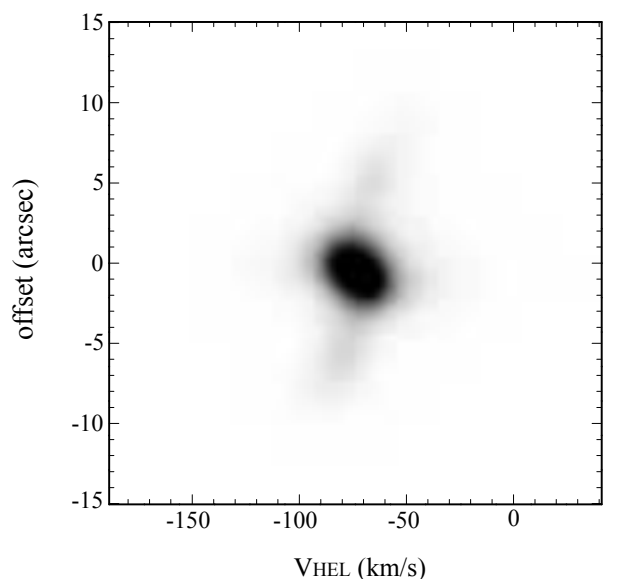

AAT - MAJOR-AXIS

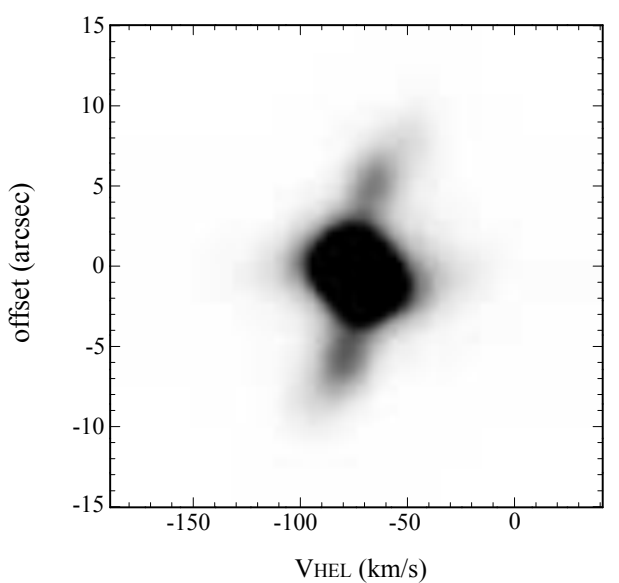

MODEL - SLIT 1

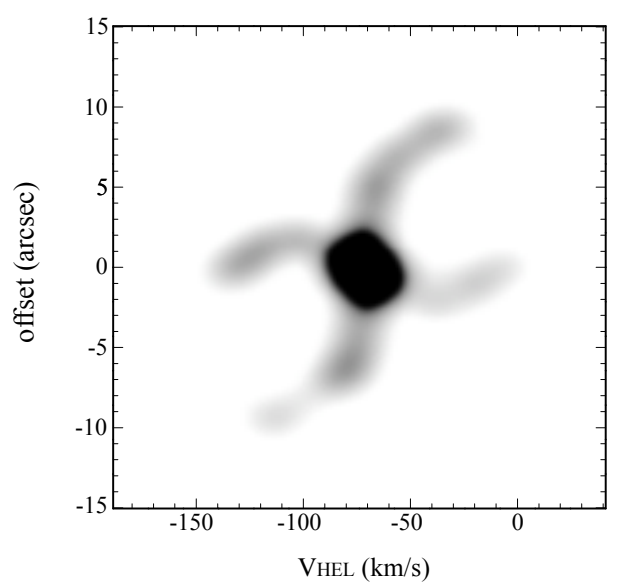

NTT - MINOR-AXIS

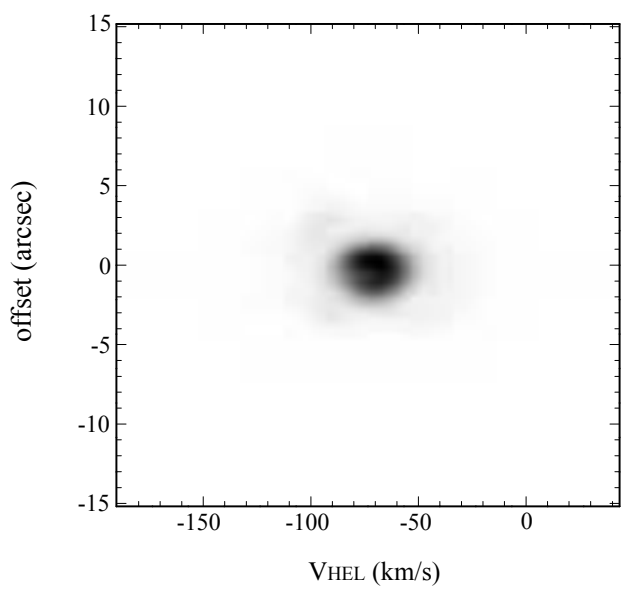

NTT - MINOR-AXIS

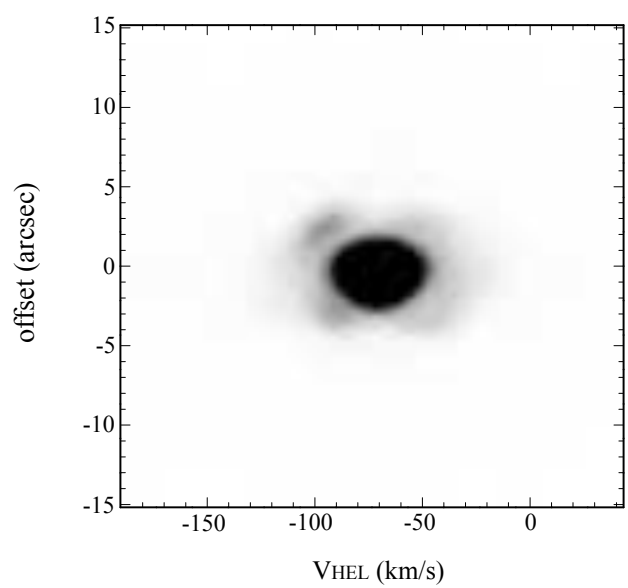

MODEL - SLIT 2

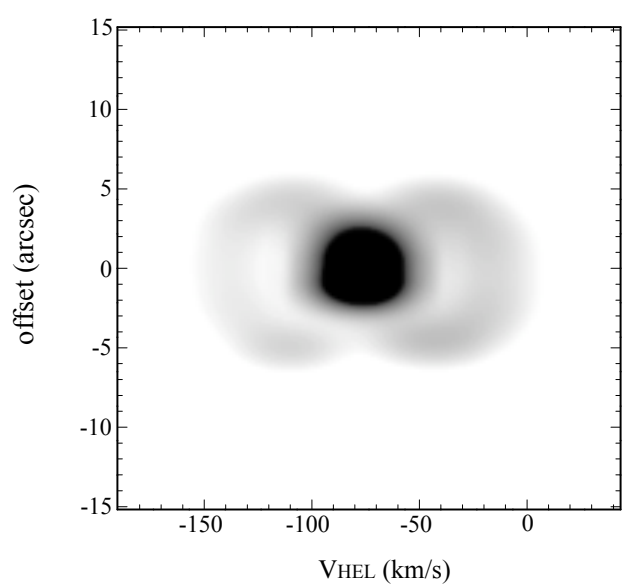

Fig. 11. MES, EMMI, and synthetic P-V arrays. The arrays on the left column (labelled AAT) are those for a long-slit applied to the major axis of the nebula using MES, whereas the right column (NTT) shows the arrays for a long-slit placed across the minor axis using EMMI (see Fig. 12b for these slit positions). The P-V arrays on the top panel and those on the central panel are the same, except using different stretches of the intensity scale. Shown at the bottom (MODEL) panel are the corresponding synthetic P-V arrays, which were generated using the SHAPE model.

MyCn 18 to form from an ILOT event, it would need to have a linear velocity-distance relation, a bipolar structure, an expansion velocity (for the knotty outflow) of $\sim 100-1000 \mathrm{~km} \mathrm{~s}^{-1}$, and a total kinetic energy of $\sim 10^{46}-10^{49} \mathrm{erg}$, as noted by Soker \& Kashi (2012). They show that MyCn 18 has all the characteristic properties of an ILOT event apart from the total kinetic energy, which is of the order $\sim 10^{44} \mathrm{erg}$, and their energy-time diagram (ETD) shows MyCn 18 to be just outside the optical transient stripe (OTS), making it an exception. MyCn 18 does not appear energetic enough to be a ILOT event. They support 

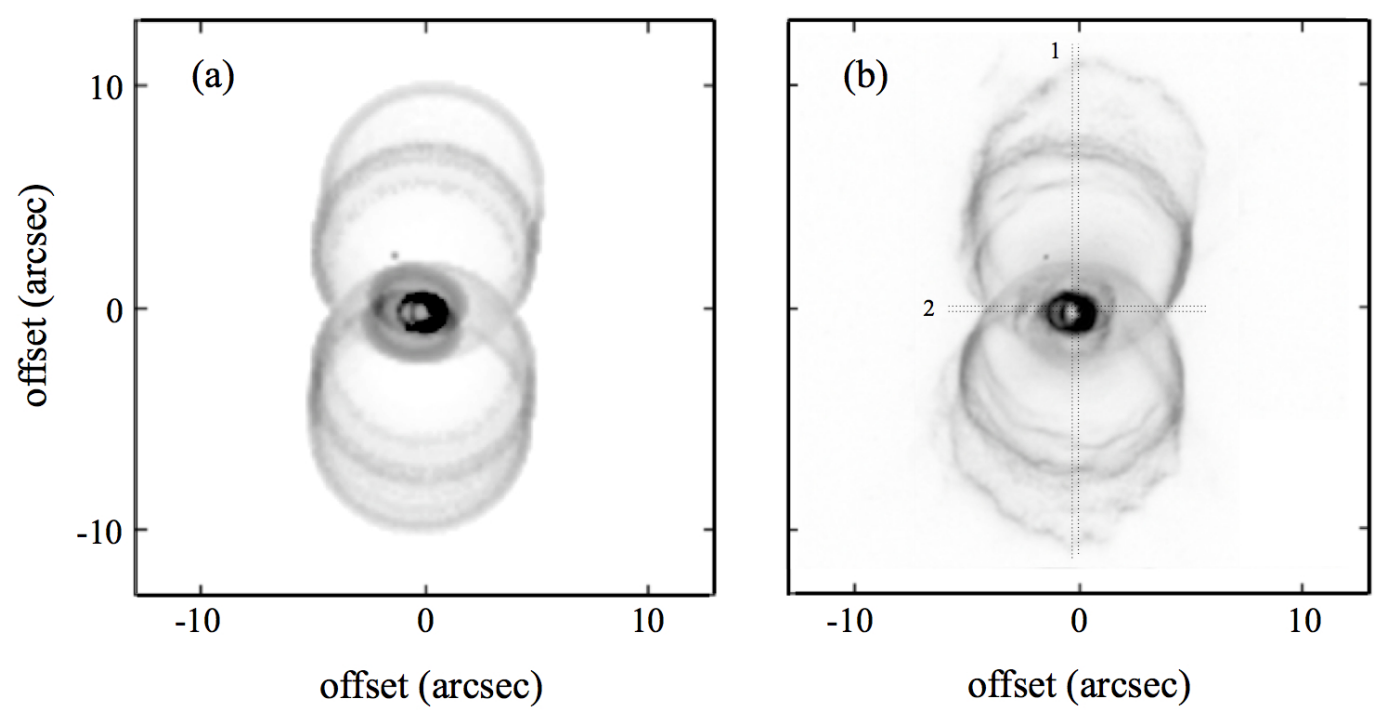

Fig. 12. The resultant SHAPE model of MyCn 18's nebular morphology and an HST[N II]6584 A image scaled for comparison. Also shown are the long-slit positions used for the observed and synthetic P-V arrays in Fig. 11, labelled 1 (slit 1 down the major-axis) and 2 (slit 2 across the minor-axis). Both slit widths have a value of 0.8 arcsec.

the O'Connor et al. (2000) view that MyCn 18 was formed by a nova like explosion instead. However, in the light of the recent frequent discovery of hot Jupiter-type planets, it is possible to generate an ILOT event of significantly lower energy than envisaged by Soker \& Kashi (2012). A planet engulfed at the AGB phase, surviving to spiral through the tenuous envelope to the dense core of the star and finally forming an accretion disk there (Nordhaus \& Blackman 2006), gives a natural mechanism for breaking the spherical symmetry of the AGB phase. Subsequently a nova-type explosion event on the degenerate core could have led to the generation of the hypersonic knots and the deviations from cylindrical symmetry of the nebula and the offset location of its central star.

A planet destruction event causing an asymmetric nova explosion could explain features as follows: on the AGB phase, a planet is engulfed and survives long enough to disrupt the symmetry of the nebula and cause its bipolar shape. As the planet spirals to the degenerate core an accretion disk forms and we speculate that the final accretion event of the planet onto the star generates an explosion that is localised on the stellar core. This asymmetric explosion gives a velocity kick to the star, generates the knotty outflow perpendicular to the disk, and causes the disruption to ring 1 in the opposite (NE) direction to the star's movement (this is observed better in the composite colour image from Sahai et al. 1999).

Interestingly, despite the expectation that planets should be readily detectable around white dwarfs (Burleigh et al. 2001) they have not been observed yet (Mustill \& Villaver 2012), which may suggest that many hot Jupiter exoplanets are readily engulfed and destroyed at the AGB phase. Since there are not enough close binaries to shape the large fraction of non-spherical $\mathrm{PNe}$, this strongly suggests some role for planets in their shaping (De Marco \& Soker 2011).

\subsection{Morpho-kinematic modelling}

Our long-slit spectra provided us with sufficient information about the 3D structure of the nebula, which allowed us to generate a self-consistent 3D model. Density distributions and velocity parameters were applied to the individual sub-systems in order to generate the synthetic P-V arrays needed for describing the kinematics of the nebula. This technique, along with the long-slit spectra, enabled us to reconstruct the shape of the nebula. The resultant 3D SHAPE model is in good agreement with that of Sahai et al. (1999); Dayal et al. (2000), specifically for the structure and kinematics of this gaseous nebula.

It has been shown, by use of the observed and synthetic P-V arrays, that the expansion rate of the nebula follows that of a Hubble-type expansion, i.e., the velocity of the ionised gas increases linearly with distance from the centre (Solf \& Ulrich 1985; Icke et al. 1989). Our values for the expansion rates correspond closely to those found by O'Connor et al. (2000). The kinematical age of MyCn 18 was then found to be $\sim 2700$ yr using these velocities. Although a distance of $3.2 \mathrm{kpc}$ was used to help determine an age for MyCn 18, it is still not accepted as a confirmed distance, therefore, other techniques are required to narrow down the possible range.

An unambiguous distance measurement requires a proper motion study of the high velocity knots of $\mathrm{MyCn} 18$ or hourglass edges. The proper motion for a given feature is $0.02 V_{100} / D_{\mathrm{kpc}} \operatorname{arcsec} \mathrm{yr}^{-1}$, where $V_{100}$ is the velocity in units of $100 \mathrm{~km} \mathrm{~s}^{-1}$ and $D_{\mathrm{kpc}}$ is the distance in units of kpc. The original HST images of MyCn 18 were taken in the mid 1990 s so displacement of the $500 \mathrm{~km} \mathrm{~s}^{-1}$ knots should be detectable in a second epoch image even if the distance to $\mathrm{MyCn} 18$ is up to $3.2 \mathrm{kpc}$. The second epoch which included the NTT EFOSC frames were analysed to determine the relative positions of the knots. Unfortunately, the exposure times were much too short to reveal any knots that might have moved.

\section{Conclusions}

We have carried out an optical and infrared study of MyCn 18 in order to understand the whole structure and dynamics of the nebula from the offset central star to the striking nebula morphology and the distant hypersonic knotty outflow. VLT IR observations of MyCn 18 were combined with earlier optical work, morpho-kinematic modelling, and a search for a close stellar binary companion. We consider that the conventional planetary nebula formation process has been significantly affected by an 

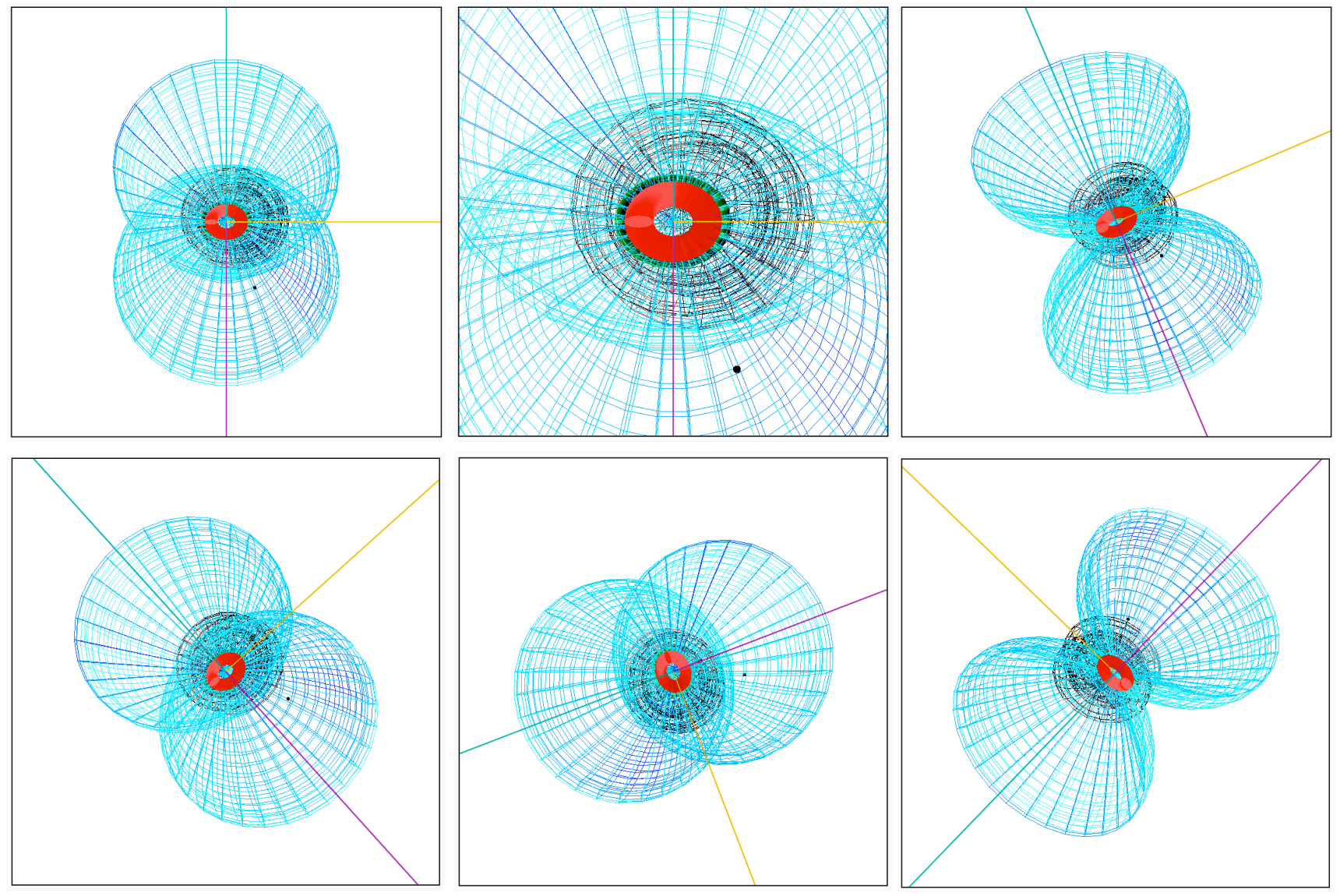

Fig. A.1. SHAPE model of MyCn 18 from different points of view.

explosive event that has led to deviations from axial symmetry such as a displacement of the central star and the misalignment of the main nebular structures. We speculate that a hot Jupiter exoplanet was engulfed at the AGB phase of the star's evolution and that the accretion of this material onto the core of the star led to a nova-type explosion that was ultimately responsible for the hypersonic knotty outflow. Given the common nature of hot Jupiters, it can even be suggested that this and other extreme bipolar morphology planetary nebulae are ultimately shaped by one or more exoplanets.

Acknowledgements. We gratefully acknowledge the support of the College of Science, NUI Galway (under their Ph.D. fellowship scheme). Based on observations collected at the European Southern Observatory, Chile, and proposal numbers are 071.D-0698 and 073.D-0359. Based on observations made with the NASA/ESA Hubble Space Telescope, obtained from the data archive at the Space Telescope Institute. STScI is operated by the association of Universities for Research in Astronomy, Inc. under the NASA contract NAS 5-26555. This paper makes use of data obtained from the Isaac Newton Group Archive which is maintained as part of the CASU Astronomical Data Centre at the Institute of Astronomy, Cambridge. We thank Eamonn Harvey for his assistance and guidance with the use of SHAPE. We also thank the anonymous referee for their thorough review and highly appreciate their comments and suggestions, which significantly contributed to improving the quality of the publication.

\section{Appendix A: SHAPE images of MyCn 18}

Shown above is the SHAPE model for MyCn 18 from different viewing angles. The green, yellow, and purple line represent the top view, right view, and axis of symmetry of the nebula, respectively. The top left view shows the nebula with its southern pole tilted towards us at an angle of $52^{\circ}$ with respect to the plane of the sky. From top to bottom on this view represents a NW to
SE direction. The view on the top middle is a close in of the first showing detail of the inner part of the nebula.

\section{References}

Aleman, I., \& Gruenwald, R. 2004, ApJ, 607, 865

Bains, I., Bryce, M., Calabretta, M., \& Stirling, A. M. 2002, MNRAS, 337, 401

Balick, B. 1987, AJ, 94, 671

Balick, B., \& Frank, A. 2002, ARA\&A, 40, 439

Bernard-Salas, J., \& Tielens, A. G. G. M. 2005, A\&A, 431, 523

Bryce, M., Lopez, J. A., Holloway, A. J., \& Meaburn, J. 1997, ApJ, 487, L161

Burleigh, M., Clarke, F., \& Hodgkin, S. 2001, in Techniques for the Detection of Planets and Life beyond the Solar System, ed. W. R. F. Dent, 15

Burton, M. G., Hollenbach, D. J., \& Tielens, A. G. G. 1992, ApJ, 399, 563

Cavanagh, B., Jenness, T., Economou, F., \& Currie, M. J. 2008, Astron. Nachr., 329,295

Chevalier, R. A., \& Luo, D. 1994, ApJ, 421, 225

Clark, D. M., López, J. A., Steffen, W., \& Richer, M. G. 2013, AJ, 145, 57

Corradi, R. L. M., \& Schwarz, H. E. 1993, A\&A, 268, 714

Cox, P., Boulanger, F., Huggins, P. J., et al. 1998, ApJ, 495, L23

Currie, M. J., \& Berry, D. S. 2000, Starlink User Note 95

Currie, M. J., \& Cavanagh, B. 2004, ORAC-DR - imaging data reduction, starlink User Note 232.8

Davis, C. J., Smith, M. D., Stern, L., Kerr, T. H., \& Chiar, J. E. 2003, MNRAS, 344,262

Dayal, A., Sahai, R., Trauger, J., et al. 1997, in BAAS, 29, 1234

Dayal, A., Sahai, R., Watson, A. M., et al. 2000, AJ, 119, 315

De Marco, O. 2009, PASP, 121, 316

De Marco, O., \& Soker, N. 2011, PASP, 123, 402

Dekker, H., Delabre, B., \& Dodorico, S. 1986, in SPIE Conf. Ser. 627, ed. D. L. Crawford, 339

Disney, M. J., \& Wallace, P. T. 1982, QJRAS, 23, 485

Douchin, D., De, M. O., Jacoby, G. H., et al. 2012, in SF2A-2012: Proc. of the Annual meeting of the French Society of Astronomy and Astrophysics, eds. S. Boissier, P. de Laverny, N. Nardetto, et al., 325 
Eyermann, S. E., \& Speck, A. K. 2004, in BAAS, 36, 138.11

García-Díaz, M. T., López, J. A., Steffen, W., \& Richer, M. G. 2012, ApJ, 761, 172

García-Segura, G., Langer, N., Różyczka, M., \& Franco, J. 1999, ApJ, 517, 767

Guerrero, M. A., Miranda, L. F., Ramos-Larios, G., \& Vázquez, R. 2013, A\&A, 551, A53

Hadrava, P. 2006, A\&A, 448, 1149

Han, Z., Podsiadlowski, P., \& Eggleton, P. P. 1995, MNRAS, 272, 800

Henney, W. J., Williams, R. J. R., Ferland, G. J., Shaw, G., \& O’Dell, C. R. 2007, ApJ, 671, L137

Hora, J. L., \& Latter, W. B. 1996, in BAAS, 28, 1402

Hora, J. L., Latter, W. B., Marengo, M., et al. 2005, in BAAS, 37, 493

Icke, V., Preston, H. L., \& Balick, B. 1989, AJ, 97, 462

Jones, D., Lloyd, M., Santander-García, M., et al. 2010, MNRAS, 408, 2312

Jones, D., Mitchell, D. L., Lloyd, M., et al. 2012, MNRAS, 420, 2271

Jordan, S., Bagnulo, S., Werner, K., \& O'Toole, S. J. 2012, A\&A, 542, A64

Kastner, J. H., Henn, L., Weintraub, D. A., Gatley, I., \& Siebenmorgen, R. 1998, in BAAS, 30, 1274

Kastner, J. H., Gatley, I., \& Weintraub, D. A. 2000, in Asymmetrical Planetary Nebulae II: From Origins to Microstructures, eds. J. H. Kastner, N. Soker, \& S. Rappaport, ASP Conf. Ser., 199, 355

Koller, J., \& Kimeswenger, S. 2000, Thermal Emission Spectroscopy and Analysis of Dust, Disks, and Regoliths, 196, 23

Kwok, S. 1982, ApJ, 258, 280

Kwok, S., Purton, C. R., \& Fitzgerald, P. M. 1978, ApJ, 219, L125

Lee, T.-H., Stanghellini, L., Ferrario, L., \& Wickramasinghe, D. T. 2007, in 15th European Workshop on White Dwarfs, eds. R. Napiwotzki, \& M. R. Burleigh, ASP Conf. Ser., 372, 173

Lenzen, R., Hartung, M., Brandner, W., et al. 2003, in SPIE Conf. Ser., 4841, eds. M. Iye, \& A. F. M. Moorwood, 944

Likkel, L., Kindt, A., Dinerstein, H. L., \& Lester, D. F. 2000, in Asymmetrical Planetary Nebulae II: From Origins to Microstructures, eds. J. H. Kastner, N. Soker, \& S. Rappaport, ASP Conf. Ser., 199, 333

Maihara, T., Iwamuro, F., Yamashita, T., et al. 1993, PASP, 105, 940

Matsuura, M., \& Zijlstra, A. 2005, in High Resolution Infrared Spectroscopy in Astronomy, eds. H. U. Käufl, R. Siebenmorgen, \& A. Moorwood, 423

Matsuura, M., Speck, A. K., Smith, M. D., et al. 2007, MNRAS, 382, 1447

Meaburn, J., Blundell, B., Carling, R., et al. 1984, MNRAS, 210, 463

Moorwood, A., Cuby, J.-G., Ballester, P., et al. 1999, The Messenger, 95, 1

Morris, M. 1987, PASP, 99, 1115

Mustill, A. J., \& Villaver, E. 2012, ApJ, 761, 121

Nordhaus, J., \& Blackman, E. G. 2006, MNRAS, 370, 2004
O’Connor, J. A., Redman, M. P., Holloway, A. J., et al. 2000, ApJ, 531, 336

Oliva, E., \& Origlia, L. 1992, A\&A, 254, 466

Pascoli, G., \& Lahoche, L. 2008, PASP, 120, 1267

Pickles, A. J. 1998, PASP, 110, 863

Ramos-Larios, G., Guerrero, M. A., \& Miranda, L. F. 2008, AJ, 135, 1441

Ramos-Larios, G., Guerrero, M. A., Suárez, O., Miranda, L. F., \& Gómez, J. F. 2012a, A\&A, 545, A20

Ramos-Larios, G., Vázquez, R., Guerrero, M. A., et al. 2012b, MNRAS, 423, 3753

Rousselot, P., Lidman, C., Cuby, J.-G., Moreels, G., \& Monnet, G. 2000, A\&A, 354, 1134

Rousset, G., Lacombe, F., Puget, P., et al. 2003, in SPIE Conf. Ser. 4839, eds. P. L. Wizinowich, \& D. Bonaccini, 140

Sahai, R., \& Trauger, J. T. 1998, AJ, 116, 1357

Sahai, R., Dayal, A., Watson, A. M., et al. 1999, AJ, 118, 468

Sahai, R., Sugerman, B. E. K., \& Hinkle, K. 2009, ApJ, 699, 1015

Schwarz, H. E., Corradi, R. L. M., \& Melnick, J. 1992, A\&AS, 96, 23

Sellgren, K., Bromm, V., \& Dinerstein, H. 2008, Spitzer Proposal, 50179

Soker, N., \& Harpaz, A. 1992, PASP, 104, 923

Soker, N., \& Kashi, A. 2012, ApJ, 746, 100

Soker, N., \& Rappaport, S. 2000, ApJ, 538, 241

Soker, N., \& Rappaport, S. 2001, ApJ, 557, 256

Solf, J., \& Ulrich, H. 1985, A\&A, 148, 274

Stanghellini, L., Shaw, R. A., \& Villaver, E. 2008, ApJ, 689, 194

Steffen, W., Koning, N., Wenger, S., Morisset, C., \& Magnor, M. 2011, IEEE Transactions on Visualization and Computer Graphics, 17, 454

Tenenbaum, E. D., Milam, S. N., Woolf, N. J., \& Ziurys, L. M. 2009, ApJ, 704 L108

Trammell, S. R., Dinerstein, H. L., \& Goodrich, R. W. 1995, in Asymmetrical Planetary Nebulae, eds. A. Harpaz, \& N. Soker, Ann. Israel Phys. Soc., 11, 37

Tyndall, A. A., Jones, D., Lloyd, M., O’Brien, T. J., \& Pollacco, D. 2012, MNRAS, 422, 1804

Vlemmings, W. 2012, in IAU Symp., 283, 176

Vlemmings, W. H. T. 2011, in Asymmetric Planetary Nebulae 5 Conf.

Volk, K. 2003, in Planetary Nebulae: Their Evolution and Role in the Universe, eds. S. Kwok, M. Dopita, \& R. Sutherland, IAU Symp., 209, 281

Wang, M.-Y., Muthumariappan, C., \& Kwok, S. 2006, in Planetary Nebulae in our Galaxy and Beyond, eds. M. J. Barlow, \& R. H. Méndez, IAU Symp., 234, 537

Whitelock, P. A. 1985, MNRAS, 213, 59

Yungelson, L. R., Tutukov, A. V., \& Livio, M. 1993, ApJ, 418, 794 